\title{
3
}

\section{Quantum Markov Semigroups}

Somigroups of completely positive operators are a fundamental tool in the theory of quantum Markov processes. Several results on this class of semigroups mostly in the uniformly continuous case - are scattered in the literature. In this chapter we give a self-cont ained exposition of the basic results on uniformly contimuous completely positive semigroups. Yforeover we describe the construction of completely positive semigroups on $\mathcal{B}(h)$ that are continuous in the $\sigma$-weak topology and we give conditions - necessary and sufficient or simply sufficient for the semigroup to be identity preserving (i.e. Markov).

\subsection{Fundamental definitions and examples}

In this section $\mathcal{A}$ will denote a $W^{*}$-algebra of operators acting on a Hilbert space $\mathcal{H}$ and $\mathcal{A}_{*}$ will denote its predual.

Definition 3.1 A quantum dynamical semigroup on $\mathcal{A}$ is a family $\mathcal{T}=\left(\mathcal{T}_{t}\right)_{t \geq 0}$ of bounded operators on $\mathcal{A}$ with the following properties:

1. $\mathcal{T}_{0}(a)=a$. for all $a \in \mathcal{A}$,

2. $\mathcal{T}_{t+s}(a)=\mathcal{T}_{t}\left(\mathcal{T}_{s}(a)\right)$, for all $s, t \geq 0$ and all $a \in \mathcal{A}$,

3. $\mathcal{T}_{t}$ is completely positive for all $t \geq 0$,

4. $\mathcal{T}_{t}$ is a $\sigma$-weakly continuous operator in $\mathcal{A}$ for all $t \geq 0$,

5. for each $a \in \mathcal{A}$, the map $t \rightarrow \mathcal{T}_{t}(a)$ is continuous with respect to the $\sigma$-weak topology on $\mathcal{A}$.

Notice that, when 3 holds, then 4 is equivalent to normality of the maps $\mathcal{T}_{t}$ by Proposition 1.15.

We introduce the usual definition of the infinitesimal generator of $\mathcal{T}$. 
Definition 3.2 The infinitesimal generator the quantum dynamical semigroup $\mathcal{T}$ is the operator $\mathcal{L}$ whose domain $D(\mathcal{L})$ is the vector space of elements a in $\mathcal{A}$ for which there exists an element $b$ of $\mathcal{A}$ such that

$$
b=\lim _{t \rightarrow 0} \frac{\mathcal{T}_{t}(a)-a}{t}
$$

in the $\sigma$-weak topology, and $\mathcal{L}(a)=b$.

We now give some examples.

Example 3.1 Let $\mathcal{A}=\mathcal{B}(\mathcal{H})$ and let $\left(P_{t}\right)_{t \geq 0}$ be a strongly continuous semigroup on $\mathcal{H}$. The family of linear operators $\overline{\mathcal{T}}_{t}: \mathcal{A} \rightarrow \mathcal{A}$ defined by

$$
\mathcal{T}_{t}(a)=P_{t}^{*} a P_{t}
$$

is a quantum dynamical semigroup. In fact all the continuity properties of $\mathcal{T}$ follow from Kraus' theorem and the strong continuity of $P$. Let $G$ denote the infinitesimal generator of $P$. The infinitesimal generator $\mathcal{L}$ of $\mathcal{T}$ is given (formally if $G$ is unbounded) by

$$
\mathcal{L}(a)=G^{*} a+a G .
$$

Notice that, if $G$ is unbounded, then $\mathcal{T}$ is not necessarily strongly continuous.

The following example shows that classical Markov semigroups can be viewed as a special class of quantum dynamical semigroups. However some care must be taken dealing with topologies.

Example 3.2 Let $(X, \mathcal{X})$ be a measurable space and let $\mu$ be a $\sigma$-finite measure on $\mathcal{X}$. Then $L^{\infty}(X . \mathscr{C} ; d \mu)$ is a commutative $W^{*}$-algebra of multiplication operators acting on the Hilbert space $L^{2}(X, \mathbb{C} ; d \mu)$. The predual is the Banach space $L^{1}\left(X, \mathbb{C}^{\prime} ; \mu\right)$.

Consider a family $(P(t, \cdot ; \cdot))_{t \geq 0}$ of transition probabilities on $X \times \mathcal{X}$ with the following properties:

1. for all $x \in X, P(t, x ; \cdot)$ is a probability measure on $\mathcal{X}$, absolutely continuous with respect to $\mu$ for $t>0$, and coinciding with the Dirac moasure $\delta_{x}$ for $t=0$,

2. for all measurable sets $A \in \mathcal{X}, P(t, \cdot ; A)$ is a measurable essentially bounded function on $X$ and the map $t \rightarrow P(t, ; A)$ defined on $[0,+\infty[$ with values in $L^{\infty}(X, \mathcal{X} ; d \mu)$ is $\sigma$-weakly continuous,

3. (Chapman-Kolmogorov equation) for all $t, s \geq 0, x \in X$ and $A \in \mathcal{X}$ we have

$$
P(t+s, x ; A)=\int_{X} P(t, y ; A) P(s, x ; d y) .
$$


Let $\left(\mathcal{T}_{t}\right)_{t \geq 0}$ be the family of operators on $L^{\infty}(X, \mathbb{C} ; d \mu)$

$$
\left(\mathcal{T}_{t} f\right)(x)=\int_{X} f(y) P(t, x ; d y) .
$$

Then $\mathcal{T}$ is a quantum dynamical semigroup in the $W^{*}$-algebra $L^{\infty}(X, \mathbb{C} ; d \mu)$. In fact property 1 of a quantum dynamical semigroup follows from $P(0, x ; d y)=$ $\delta_{x}(d y)$ and property 2 follows from the Chapman-Kolmogorov equation. Complete positivity follows from the fact that the $W^{*}$-algebra $L^{\infty}(X, \mathbb{C} ; d \mu)$ is commutative and that the linear operators $\mathcal{T}_{t}$ are positive.

The continuity property 5 follows from the $\sigma$-weakly continuity of the map $t \rightarrow P(t, ; A)$ for every $A \in \mathcal{X}$. Indeed, writing $f$ as the sum four positive functions and remembering that the $\sigma$-weak and weak topology coincide on bounded subsets of $L^{\infty}(X, \mathbb{C} ; d \mu)$, we can easily see that it suffices to show that, for wery positive element $f$ of $L^{\alpha}(X, \mathbb{C} ; d \mu)$ and every $y \in L^{2}(X, \mathbb{C} ; d \mu)$, the function

$$
t \rightarrow\left\langle g(\cdot), \int_{X} f(y) P(t, \cdot ; d y) g(\cdot)\right\rangle=\int_{X}|g(x)|^{2} d \mu(x) \int_{X} f(y) P(t, x ; d y)
$$

is continuous. This is clearly the case whenever $f$ is a simple function because of the $\sigma$-weakly continuity of the maps $t \rightarrow P(t, \therefore A)$ with $A \in \mathcal{X}$. Approximating $f$ by an increasing (resp. decreasing) sequence of simple functions converging to $f$ almost everywhere we see that (3.1) is lower (resp. upper) semicontinuous. Therefore the function (3.1) is continuous.

In order to prove the continuity property 4 , since $\mathcal{T}_{t}$ is positive, by Proposition 1.15 it suffices to check that it is normal. Thus we must show that. for every increasing net $\left(f_{\mathrm{a}}\right)$ in $L^{1}(X, \mathbb{C} ; d \mu)$ converging $\sigma$-weakly to $f$ in $L^{1}(X, \mathbb{C} ; d \mu)$ and every $g \in L^{1}(X, \mathscr{C} ; d \mu)$ non-negative we have

$$
\sup _{n} \int_{X} g(x) d \mu(x) \int_{X} f_{\alpha}(y) P(t, x ; d y)=\int_{X} g(x) d \mu(x) \int_{X} f(y) P(t, x ; d y) .
$$

By [21] Lemma 2.4 .19 p.76, the net $\left(f_{\alpha}\right)$ converges $\sigma$-strongly to $f$. Therefore, denoting the Radon-Nikodym derivative of $P(t, x: d y)$ with respect to $\mu$ by $p(t, x, \cdot)$, we have

$$
\begin{aligned}
\sup _{\alpha} \int_{X} f_{\alpha}(y) P(t, x ; d y) & =\sup _{\alpha}\left\langle(p(t, x, \cdot))^{1 / 2}, f_{\alpha}(\cdot)(p(t, x, \cdot))^{1 / 2}\right\rangle \\
& =\left\langle(p(t, x \cdot \cdot))^{1 / 2}, f(\cdot)(p(t, x, \cdot))^{1 / 2}\right\rangle \\
& =\int_{X} f(y) P(t . x ; d y)
\end{aligned}
$$

for every $x \in X$. It follows that the right-hand side is the least upper bomd of the integrals of $f_{\alpha}$ with respect to $P(t, x ; d y)$ and the same argument yields (3.2). This shows that $\left(\mathcal{T}_{t}\right)_{t \geq 0}$ is a quantum dymamical semigroup. 
It is worth noticing that a large class of classical Markov semigroups enjoys the so-called Feller property. This means, roughly speaking, that the semigroup can be restricted to a space of continuous functions and studied as a strongly continuous contractive semigroup. The original semigroup on $L^{\infty}(X, \mathcal{X} ; \mu)$ is then the unique $\sigma$-weakly continuous extension of this restriction. Clearly the quantum analogue of the Feller property is the following: there exists a $C^{*}$ algebra $\mathcal{A}_{0}$ that is dense in $\mathcal{A}$ in the $\sigma$-weak operator topology, invariant under the action of the quantum dynamical semigroup $\mathcal{T}$, and such that the restriction of $\mathcal{T}$ to $\mathcal{A}_{0}$ is strongly continuous. We will not be concerned however with the problem of establishing whether some quantum dynamical semigroup enjoys this property.

The following fact will be useful in the construction of another example of a quantum dynamical semigroup through a generalization of Example 3.1

Proposition 3.3 Let $(X, \mathcal{X})$ be a measurable space and let $\mu$ a finite measure on $\mathcal{X}$. Let $(U(t, x))_{t \geq 0, x \in X}$ be a family of bounded operators on a Hilbert space $\mathcal{H}$ such that

1. for all $x \in X$ the map $t \rightarrow U(t, x)$ is strongly continuous,

2. for all $t \geq 0$ the map $x \rightarrow U(t, x)$ is strongly musurable,

3. for all $t \geq 0$ there exists a positive function $g_{t}$ on $X$, integrable with respect to $\mu$, such that

$$
\sup _{0 \leq s \leq l}\|U(s, x)\| \leq g_{t}(x)
$$

Then the map $\Phi:[0,+\infty[\times \mathcal{B}(\mathcal{H}) \rightarrow \mathcal{B}(\mathcal{H})$ defined by the integral (in the $\sigma$-weak topology)

$$
\Phi(t, a)=\int_{X} U(t, x)^{*} a U(t, x) d \mu(x)
$$

is $\sigma$-weakly continuous in both arguments and completely positive in the second.

Proof. Let $n \geq 1$ and $a_{1}, \ldots, a_{n}, b_{1}, \ldots, b_{n}$ be clements of $\mathcal{B}(\mathcal{H})$. Since

$$
\sum_{i, j=1}^{n} b_{j}^{*} \Phi\left(t, a_{j}^{*} a_{i}\right) b_{i}=\int_{X}\left|\sum_{j=1}^{n} a_{j} U(t, x) b_{j}\right|^{2} d \mu(x) \geq 0
$$

the map $\Phi(t, \cdot)$ is completely positive by Proposition 2.8 .

Note that $\Phi$ is uniformly bounded on sets of the form $[0, t] \times \mathcal{B}(\mathcal{H})$ with $t$ fixed. Therefore, since the $\sigma$-weak and weak topology coincide on bounded sets of $\mathcal{B}(\mathcal{H})$, in order to show that $\Phi$ is $\sigma$-weakly continuous in a it suffices to prove that, for every $u \in \mathcal{H}$, the positive linear functional on $\mathcal{B}(\mathcal{H})$

$$
a \rightarrow \int_{X}\langle U(t, x) u, a U(t . x) u\rangle d \mu(x)
$$


is $\sigma$-weakly continuous. To this end, by [21] Th. 2.4 .21 p.76, it suffices to show that, for every increasing net $\left(a_{\alpha}\right)$ in $\mathcal{B}(\mathcal{H})$ with least upper bound $a$ we have

$$
\sup _{\alpha} \int_{X}\left\langle U(t, x) u, a_{\alpha} U(t, x) u\right\rangle d \mu(x)=\int_{X}\langle U(t, x) u, a U(t, x) u\rangle d \mu(x) .
$$

Since $\left(a_{\alpha}\right)$ converges strongly to $a$ (see [21] Lemma 2.4.19) for all $x \in X$, we have

$$
\sup _{\alpha}\left\langle U(t, x) u, a_{\alpha} U(t, x) u\right\rangle=\langle U(t, x) u, a U(t, x) u\rangle .
$$

The conclusion then follows since the map on $L^{1}(X, \mathcal{X}, \mu)$

$$
g \rightarrow \int_{X} g(x) d \mu(x)
$$

is it $\tau$-weakly continuous functional. (This is in practice a monotone convergence theorem for increasing nets in $L^{1}(X, \mathcal{X}, \mu)$ ).

Finally to show that $\Phi$ is $\sigma$-weakly continuous in $t$ it suffices to use the inequality

$$
|\langle u,(\Phi(t, a)-\Phi(s, a)) u\rangle| \leq 2 c(r)\|a\|\|u\| \int_{X}\|(U(t, x)-U(s, x)) u\| d \mu(x)
$$

for $u \in \mathcal{H}$ and $t, s \in[0, r]$ ( $r>0$ fixed) where $c(r)$ is a positive constant depending only on $r$ and apply Lebesgue's theorem.

The following example is a generalization of Example 3.1 due to Parthasarathy ([74] Example 30.1 p. 258). However the semigroup he constructs there is not a quantum dynamical semigroup in the sense of his definition (Sect. 30 p.257) since it is not necessarily strongly continuous. We shall exhibit a counterexample.

Example 3.3 Let $\left(B_{t}\right)_{t \geq 0}$ be a classical Brownian motion on a filtered probability space $(\Omega, \mathcal{F}, \mathbb{P})$ and let $\mathcal{H}$ be a complex separable Hilbert space. For every bounded self-adjoint operator $L$ on $\mathcal{H}$ let us define the map $\mathcal{T}_{t}: \mathcal{B}(\mathcal{H}) \rightarrow \mathcal{B}(\mathcal{H})$

$$
\begin{aligned}
\mathcal{T}_{t}(a) & =\int_{\Omega} \exp \left(i L B_{t}(\omega)\right) a \exp \left(-i L B_{t}(\omega)\right) d \mathbb{P}(\omega) \\
& =\frac{1}{\sqrt{2 \pi}} \int_{\mathbb{R}} \exp (i x \sqrt{t} L) a \exp (-i x \sqrt{t} L) \exp \left(-\frac{x^{2}}{2}\right) d x
\end{aligned}
$$

Since $\left(B_{t}\right)_{t \geq 0}$ has stationary independent increments it can be shown as in [74] Example 30.1 p. 258 that $\left(\mathcal{T}_{t}\right)_{t \geq 0}$ is a semigroup. Applying Proposition 3.3 with $U(t, x)=\exp (-i x \sqrt{t} L)$ and $\mu$ equal to the standard gaussian measure on the Borel $\sigma$-algebra of $\mathbb{R}$ it is easy to see that also the properties 3, 4 and 5 of Definition 3.1 are fulfilled. Hence $\mathcal{T}$ is a quantum dynamical semigroup.

The infinitesimal generator $\mathcal{L}$ of $\mathcal{T}$ is given (at least formally if $L$ is unbounded) by

$$
\mathcal{L}(X)=-\frac{1}{2}\left(L^{2} X-2 L X L+X L^{2}\right) .
$$


The quantum dynamical semigroup $\mathcal{T}$ admits restrictions to abelian subalgebras of $\mathcal{B}(\mathcal{H})$ which are classical Markov semigroups. Our argument here is only formal since the operator $L$ is unbounded; we will rigorously deal with this example later.

Let $\mathcal{H}$ be the Hilbert space $L^{2}(\mathbb{R} ; \mathbb{C})$ and let $L$ be the first derivative with its natural domain. Denote by $M(f)$ the multiplication operator by a bounded smooth function $f$

$$
M(f): \mathcal{H} \rightarrow \mathcal{H}, \quad(M(f) u)(x)=f(x) u(x) .
$$

A straightforward computation shows that

$$
\mathcal{L}(M(f))=M\left(\frac{1}{2} f^{\prime \prime}\right)
$$

This means that the restriction of the semigroup $\mathcal{T}$ to the abelian $C^{*}$-algebra of bounded complex-valued continuous functions on the real line coincides with the classical Markov semigroup of Brownian motion.

We give now the counterexample showing that $\mathcal{T}$ is not necessarily strongly continuous.

Let us fix $\mathcal{H}=l^{2}(\mathrm{~N})$ with canonical orthonormal basis $\left(e_{n}\right)_{n \geq 0}$ and let $N$ and $S$ be the number operator and right shift operator

$$
\begin{array}{ll}
D(N)=\left\{\left.u \in \mathcal{H}\left|\sum_{n \geq 0} n^{2}\right| u_{n}\right|^{2}<+\infty\right\}, & \text { vu } u=\sum_{n \geq 0} n u_{n} e_{n} \\
D(S)=\mathcal{H}, & S u=\sum_{n \geq 0} u_{n} e_{n+1}
\end{array}
$$

where $u=\sum_{n \geq 0} u_{n} e_{n}$. Clearly $N^{2}$ is the self-adjoint operator

$$
D\left(N^{2}\right)=\left\{\left.u \in \mathcal{H}\left|\sum_{n \geq 0} n^{4}\right| u_{n}\right|^{2}<+\infty\right\}, \quad N u^{2}=\sum_{n \geq 0} n^{2} u_{n} e_{n}
$$

Let $L=N^{2}$. We now prove that the quantum dynamical semigroup $\mathcal{T}$ is not strongly continuous on $\mathcal{B}(\mathcal{H})$.

By virtue of (3.3), for every $u \in \mathcal{H}$ and $x \in \mathcal{B}(\mathcal{H})$ we have

$$
\begin{aligned}
& \left\langle u,\left(\mathcal{T}_{t}(x)-x\right) u\right\rangle \\
= & \frac{1}{\sqrt{2 \pi t}} \int_{\mathbb{R}}\left\langle u,\left(\exp \left(i y N^{2}\right) x \exp \left(-i y N^{2}\right)-x\right) u\right\rangle \exp \left(-\frac{y^{2}}{2 t}\right) d y \\
= & \frac{1}{\sqrt{2 \pi}} \int_{\mathbb{R}}\left\langle u,\left(\exp \left(i y \sqrt{t} N^{2}\right) x \exp \left(-i y \sqrt{t} N^{2}\right)-x\right) u\right\rangle \exp \left(-\frac{y^{2}}{2}\right) d y
\end{aligned}
$$


Now let $x$ be the right shift operator $S$ and, for every $n \geq 1$, let $v_{n}$ be the vector

$$
v_{n}=\frac{1}{\sqrt{n}} \sum_{n<k \leq 2 n} e_{k}
$$

We have then, for every $k \geq 1$,

$$
\exp \left(i y \sqrt{t} N^{2}\right) S \exp \left(-i y \sqrt{t} N^{2}\right) e_{k}=\exp (i y \sqrt{t}(2 k+1)) e_{k+1} .
$$

Hence we have

$$
\begin{aligned}
& \left\langle v_{n,},\left(\mathcal{T}_{t}(S)-S\right) v_{n}\right\rangle \\
= & \frac{1}{n \sqrt{2 \pi}} \int_{\mathbb{R}} \sum_{n<j, k \leq 2 n}\left\langle e_{j},\left(\exp (i y \sqrt{t}(2 k+1)-1) e_{k+1}\right\rangle \mathrm{e}^{-y^{2} / 2} d y\right. \\
= & \frac{1}{n} \sum_{n+1<k \leq 2 n} \frac{1}{\sqrt{2 \pi}} \int_{\mathbb{R}}\left(\exp (i y \sqrt{t}(2 k+1)-1) \exp \left(-\frac{y^{2}}{2}\right) d y\right. \\
= & \frac{1}{n} \sum_{n+1<k \leq 2 n}\left(\exp \left(-t(2 k+1)^{2} / 2\right)-1\right)
\end{aligned}
$$

Therefore we obtain the inequalities

$$
\begin{aligned}
\left|\left\langle v_{n},\left(\mathcal{T}_{t}(S)-S\right) v_{n}\right\rangle\right| & =\frac{1}{n} \sum_{n+1<k \leq 2 n}\left(1-\exp \left(-t(2 k+1)^{2} / 2\right)\right) \\
& \geq \frac{1}{n} \sum_{n+1<k \leq 2 n}\left(1-\exp \left(-t(2 n+5)^{2} / 2\right)\right) \\
& =\frac{n-1}{n}\left(1-\exp \left(-t(2 n+5)^{2} / 2\right)\right)
\end{aligned}
$$

This implies that

$$
\left\|\mathcal{T}_{t}(S)-S\right\| \geq \limsup _{n \rightarrow \infty}\left|\left\langle v_{n},\left(\mathcal{T}_{l}(S)-S\right) v_{n}\right\rangle\right|=1
$$

for every $t>0$ and shows that $t$ is not strongly continuous.

The above examples and the analogy with classical probability motivate the following

Definition 3.4 A quantum dynamical semigroup is called Markov or conservative or identity preserving (resp. sub-Markov) if

$$
\mathcal{T}_{t}(\mathbb{1})=\mathbb{1}, \quad\left(\operatorname{resp} \cdot \mathcal{T}_{t}(\mathbb{1}) \leq \mathbb{1}\right)
$$

for every $t \geq 0$. 
Quantum dynamical semigroup can be viewed as dual semigroups of strongly continuous semigroups on the predual Banach space $\mathcal{A}_{*}$.

Definition 3.5 The predual semigroup of a quantum dynamical semigroup $\mathcal{T}$ on $\mathcal{A}$ is the semigroup $\mathcal{S}$ of operators in $\mathcal{A}_{*}$ defined by

$$
\left(\mathcal{S}_{t}(\omega)\right)(a)=\omega\left(\mathcal{T}_{t}(a)\right)
$$

for every $a \in \mathcal{A}$ and every $\omega \in \mathcal{A}_{*}$.

Since $\mathcal{T}$ is continuous with respect to the weak topology on $\mathcal{A}$ the semigroup $\mathcal{S}$ is also contimuous with respect to the weak topology on the Bariach space $\mathcal{A}_{*}$. Therefore, by a well-known fact (see, for instance [21] Cor. $3.1 .8 \mathrm{p} .168$ ), $\mathcal{S}$ is a strongly continuous semigroup in the Banach space $\mathcal{A}_{*}$. Moreover the following are well-known results in semigroup theory:

Proposition 3.6 Let $\mathcal{T}$ be a quantum dynamical semigroup on $\mathcal{A}$ then:

1. there exists two real numbers $M, \beta$ such that.

$$
\left\|\mathcal{T}_{t}\right\| \leq M \exp (\beta t), \quad t \geq 0,
$$

2. the infinitesimal generator $\mathcal{L}$ is densely defined and closed for the $\sigma$-weak topology,

3. if she $\lambda>\beta$ then the range of $\lambda \mathbb{H}-\mathcal{L}$ coincides with $\mathcal{A}$ and we have the inequality

$$
\left\|(\lambda \mathbb{1}-\mathcal{L})^{-1}(a)\right\| \leq \frac{M}{\Re e \lambda-\beta}\|a\|,
$$

4. the resolvent operator $(\lambda \mathbb{1}-\mathcal{L})^{-1}$ is given by the Laplace transform

$$
(\lambda \mathbb{1}-\mathcal{L})^{-1}(a)=\int_{0}^{\infty} d t \exp (-\lambda t) \mathcal{T}_{i}(a)
$$

for every $a \in \mathcal{A}$ and every complex number $\lambda$ with 解 $\lambda>\beta$.

Proof. We refer to Prop. 3.1.6 p. 166 in [21].

\subsection{Uniformly continuous QDS}

Uniformly continuous quantum dynarnical semigroups already form an interesting class, in contrast to the case of elassical uniformly continuous semigroups. We refer to the book $[10]$ for several plysical examples.

Definition 3.7 A quantum dynamícal semigroup is called uniformly continuous if

$$
\lim _{l \rightarrow 0}\left\|T_{l}-T_{0}\right\|=0
$$


The following proposition summarizes the well-known results on uniformly continuous semigroups

Proposition 3.8 Let $\mathcal{T}$ be a semigroup of bounded operators on a Banach space $E$. The following conditions are equivalent:

1. the map $t \rightarrow \mathcal{T}_{t}$ is uniformly continuous,

2. the map $t \rightarrow \mathcal{T}_{t}$ is uniformly differentiable,

3. the infinitesimal generator $\mathcal{L}$ is a bounded operator and

$$
\mathcal{T}_{t}=\sum_{n \geq 0} \frac{t^{n}}{n !} \mathcal{L}^{n}
$$

the series being uniformly convergent for every real number $t$.

If these conditions are fulfilled then $\mathcal{T}$ can be extended to a uniformly continuous group of operators on $E$ satisfying

$$
\left\|\mathcal{T}_{t}\right\| \leq \exp (|t|\|\mathcal{L}\|)
$$

We refer to [21] Prop. 3.1.1 p. 161 for the proof. Notice that, if the infinitesimal generator is bounded, then $\mathcal{T}$ can be extended as a group of bounded operators in $E$. The operator $\mathcal{T}_{t}(\mathbb{1})$ is invertible for $t$ in a neighbourhood of 0 . Indeed we have the inequality

$$
\left\|\mathcal{T}_{L}(\mathbb{1})-\mathbb{1}\right\| \leq|t| \cdot\|\mathcal{L}\| \exp (|t| \cdot\|\mathcal{L}\|) .
$$

The right-hand side is smaller than one for $t$ in a neighbourhood of 0 . Therefore the inverse of $\mathcal{T}_{t}(\mathbb{1})$ can be defined by von Neumann's series

$$
\left(\mathcal{T}_{t}(\mathbb{1})\right)^{-1} \doteq \sum_{n=0}^{\infty}\left(\mathbb{1}-\mathcal{T}_{t}(\mathbb{1})\right)^{n}
$$

It then follows that the map $t \rightarrow\left(\mathcal{T}_{t}(\mathbb{1})\right)^{-1}$ is uniformly differentiable in a neighbourhood of 0 and

$$
\frac{d}{d t}\left(\mathcal{T}_{t}(\mathbb{1})\right)^{-1}=-\left(\mathcal{T}_{t}(\mathbb{1})\right)^{-1} \mathcal{T}_{t}(\mathcal{L}(\mathbb{1}))\left(\mathcal{T}_{t}(\mathbb{1})\right)^{-1} .
$$

Proposition 3.9 Let $\mathcal{T}$ be a uniformly continuous semigroup of bounded operators $\mathcal{T}_{i}$ in a von Neumann algebra $\mathcal{A}$ with infinitesimal generator $\mathcal{L}$. The following conditions are equivalent:

1. $\mathcal{T}_{t}$ is $\sigma$-weakly continuous for every $t \geq 0$,

2. $\mathcal{L}$ is $\sigma$-weakly continuous. 
Proof. The statement clearly follows from Proposition 3.8 and the fact that the set of $\sigma$-weakly continuous operators on $\mathcal{A}$ is norm closed.

To show this notice first that a linear niap $T$ on $\mathcal{A}$ is $\sigma$-weakly continuous if and only if, for every $\sigma$-weakly continuous linear functional $\omega$ on $\mathcal{A}$, the linear functional $a \rightarrow \omega(T a)$ is $\sigma$-weakly continuous. The set of $\sigma$-weakly conitinuous linear functionals on $\mathcal{A}$ (the predual $\mathcal{A}$ * of $\mathcal{A}$ ), however, is a norm closed subspace of the dual Banach space of $\mathcal{A}$ by Proposition 2.4 .18 p.75 [21]. Moreover, for every sequence $\left(T_{n}\right)_{n \geq 0}$ of $\sigma$-weakly continuous operators on $\mathcal{A}$ converging in norm to $T$, we have

$$
\sup _{\|a\| \leq 1}\left\|\omega\left(T_{n} a\right)-\omega(T a)\right\| \leq\|\omega\| \cdot\left\|T_{n}-T\right\|,
$$

i.e. the sequence $\left(\omega\left(T_{n}(0)\right)_{n \geq 0}\right.$ of $\sigma$-iweakly continuous linear functionals $\mathcal{A}$ is convergent in norm. This completes the proof.

The following property of uniformly continuous groups in $\mathcal{A}$ turus out to be useful often. It was proved in [35] (Cor.3, p. 210). Here we give a slightly different proof.

Proposition 3.10 Let $\mathcal{L}$ be a bounded operator on a von Neumann algebra $\mathcal{A}$ such that $\mathcal{L}\left(a^{*}\right)=(\mathcal{L}(a))^{*}$ for every $a \in \mathcal{A}$. The following conditions are equivalent:

1. for all $a \in \mathcal{A}$ and $t \geq 0$ we have

$$
\exp (t \mathcal{L})\left(a^{*}\right) \exp (t \mathcal{L})(a) \leq \exp (t \mathcal{L})\left(a^{*} a\right)
$$

2. for all $a \in \mathcal{A}$ we have

$$
a^{*} \mathcal{L}(a)+\mathcal{L}\left(a^{*}\right) a \leq \mathcal{L}\left(a^{*} a\right)
$$

Proof. Condition 2. clearly follows from 1. by differentiating at $t=0$.

We prove the converse. As a first step notice that for every $a, b \in \mathcal{A}$ such that $a b=0$, from (3.8), we have

$$
b^{*} \mathcal{L}\left(a^{*} a\right) b \geq b^{*} a^{*} \mathcal{L}(a) b+b^{*} \mathcal{L}\left(a^{*}\right) a b=(a b)^{*} \mathcal{L}(a) b+b^{*} \mathcal{L}\left(a^{*}\right)(a b)=0 .
$$

Then we prove that the operator $(\lambda-\mathcal{L})^{-1}$ is non-negative for every $\lambda>\|\mathcal{L}\|$. To this end it is enough to show that if $a$ is a self-adjoint element of $\mathcal{A}$ sucli that $(\lambda-\mathcal{L})(a)$ is non-regative, then $a$ is non-negative. Let $a=x-y$ be the decomposition of $a$ into its positive and negative parts. Clearly $x y=0$. Thus, by the above remark, we have $y \mathcal{L}(x) y \geq 0$. Therefore we have

$$
\begin{aligned}
0 & \leq y\left(\left(1-\lambda^{-1} \mathcal{L}\right)(a)\right) y \\
& =y a y-y\left(\lambda^{-1} \mathcal{L}\right)(a) y \\
& =-y^{3}-\lambda^{-1} y \mathcal{L}(x) y+\lambda^{-1} y \mathcal{L}(y) y \\
& \leq-y^{3}+\lambda^{-1} y \mathcal{L}(y) y
\end{aligned}
$$


Hence $0 \leq y^{3} \leq \lambda^{-1} y \mathcal{L}(y) y$. Thus we obtain the inequality $\|y\|^{3} \leq \lambda^{-1}\|\mathcal{L}\| \cdot\|y\|^{3}$. This implies $y=0$ since $\lambda^{-1}\|\mathcal{L}\|<1$ and $a=x$ is non-negative.

It follows that $\exp (t \mathcal{L})$ is non-negative for every $t>0$ because

$$
\exp (t \mathcal{L})=\lim _{n \rightarrow \infty}(\mathbb{1}-(t / n) \mathcal{L})^{-n}
$$

We can show now that the inequality (3.7) holds. Let $t \geq 0$ fixed. Using condition 2. and positivity of $\exp ((t-s) \mathcal{L})$ we have

$$
\begin{aligned}
& \frac{d}{d s} \exp ((t-s) \mathcal{L})\left(\exp (s \mathcal{L})\left(a^{*}\right) \exp ((s \mathcal{L})(a))\right. \\
& \quad=\exp ((t-s) \mathcal{L})\left[-\mathcal{L}\left(\exp (s \mathcal{L})\left(a^{*}\right) \exp (s \mathcal{L})(a)\right)\right. \\
& \left.+\quad \mathcal{L}\left(\exp (s \mathcal{L})\left(a^{*}\right)\right) \exp (s \mathcal{L})(a)+\exp (s \mathcal{L})\left(a^{*}\right) \mathcal{L}(\exp (s \mathcal{L})(a))\right] \\
& \leq 0
\end{aligned}
$$

Integrating this inequality on $[0, t](t \geq 0)$ we obtain the inequality (3.7).

The remaining part of this section will be devoted to the study of the structure of the infinitesimal generator of a quantum dynamical semigroup which is already revealed by the examples in the previous section.

We introduce first a notion related to complete positivity.

Definition 3.11 A bounded linear operator $\mathcal{L}$ on a von Neumann algebra $\mathcal{A}$ is called conditionally completely positive if for every integer $n \geq 1$, the linear map $\mathcal{L}^{(n)}$ on $\mathcal{A} \otimes \mathcal{M}_{n}$ defined by $\mathcal{L}^{(n)}\left(a \otimes E_{i j}\right)=\mathcal{L}(a) \otimes E_{i j}(1 \leq i, j \leq n)$ satisfies the inequality

$$
\mathcal{L}^{(n)}\left(x^{*} x\right)-x^{*} \mathcal{L}^{(n)}(x)-\mathcal{L}^{(n)}\left(x^{*}\right) x+x^{*} \mathcal{L}^{(n)}(\mathbb{1}) x \geq 0
$$

for every $x \in \mathcal{A} \otimes \mathcal{M}_{n}$.

The infinitesimal generator of a quantum dynamical semigroup is characterised by conditional complete positivity.

Proposition 3.12 Let $\mathcal{T}$ be a uniformly continuous semigroup on a von Neumann algebra $\mathcal{A}$ with infinitesimal generator $\mathcal{L}$. Then $\mathcal{T}_{t}$ is completely positive for every $t \geq 0$ if and only if $\mathcal{L}$ is conditionally completely positive and $\mathcal{L}\left(a^{*}\right)=(\mathcal{L}(a))^{*}$ for every $a \in \mathcal{A}$.

Proof. Let $\mathcal{T}$ be a quantum dynamical semigroup. With the notation introduced in Section 2.2 the semigroup $\left(\mathcal{T}_{t}^{(n)}\right)_{t \geq 0}$ is a uniformly continuous semigroup in $\mathcal{A} \otimes \mathcal{M}_{n}$. Therefore the Schwarz inequality (2.8) in Proposition 2.10

$$
\mathcal{T}_{t}^{(n)}\left(x^{*}\right)\left(\mathcal{T}_{t}^{(n)}(\mathbb{1})\right)^{-1} \mathcal{T}_{t}^{(n)}(x) \leq \mathcal{T}_{t}^{(n)}\left(x^{*} x\right)
$$

holds for every $x \in \mathcal{A} \otimes \mathcal{M}_{n}$ and every $t \geq 0$. Differentiating at $t=0$ (using (3.6)) we see that $\mathcal{L}^{(n)}$ satisfies (3.9). Hence $\mathcal{L}$ is conditionally completely positive. Moreover $\mathcal{L}\left(a^{*}\right)=(\mathcal{L}(a))^{*}$ because $\mathcal{T}_{t}\left(a^{*}\right)=\left(\mathcal{T}_{l}(a)\right)^{*}$ for $t \geq 0$. 
In order to prove the converse we may assume first that $\mathcal{L}(\mathbb{1 l}) \leq 0$. Indeed, if this is not the case, it suffices to consider the operator $\mathcal{L}_{1}=\mathcal{L}-c$ where $c=\|\mathcal{L}(\mathbb{1})\|$. Note that the left-hand side of (3.9) is the same for both $\mathcal{L}$ and $\mathcal{L}_{1}$ and that

$$
\mathcal{T}_{t}=\mathrm{e}^{c t} \exp \left(t \mathcal{L}_{1}\right) .
$$

Therefore $\mathcal{T}_{t}$ is completely positive if and only if $\exp \left(t \mathcal{L}_{1}\right)$ is.

So assume $\mathcal{L}(\mathbb{1}) \leq 0$, then for every $n \geq 1$, the inequality (3.9) yields

$$
x^{*} \mathcal{L}^{(n)}(x)+\mathcal{L}^{(n)}\left(x^{*}\right) x \leq \mathcal{L}^{(n)}\left(x^{*} x\right) .
$$

Therefore Proposition 3.10 implies that the operators $\mathcal{T}_{t}^{(n)}$ are positive i.e. the operators $\mathcal{T}_{t}$ are completely positive.

Lemma 3.13 Let $\mathcal{L}$ be a conditionally completely positive operator on a von Neumann algebra $\mathcal{A}$. Then for every integer $n \geq 1$, every family $a_{1}, \ldots, a_{n}$ of elements of $\mathcal{A}$ and every family $u_{1}, \ldots, u_{n}$ of vectors in $\mathcal{H}$ such that

$$
\sum_{j=1}^{n} a_{j} u_{j}=0
$$

we have

$$
\sum_{i, j=1}^{n}\left\langle u_{i}, \mathcal{L}\left(a_{i}^{*} a_{j}\right) u_{j}\right\rangle \geq 0 .
$$

Proof. Considering the elenient $x=\sum_{j} a_{j} \nsim E_{j}$ in $\mathcal{A} \nsim \mathcal{M}_{n}$ and the vector $u=\left(u_{1}, \ldots, u_{n}\right)$ in the direct sum of $n$ copies of $\mathcal{H}$ wc have

$$
\begin{aligned}
0 & \leq\left\langle u,\left(\mathcal{L}^{(n)}\left(x^{*} x\right)-x^{*} \mathcal{L}^{(n)}(x)-\mathcal{L}^{(n)}\left(x^{*}\right) x+x^{*} \mathcal{L}^{(n)}(\mathbb{1}) x\right) u\right\rangle \\
& =\sum_{i, j=1}^{n}\left\langle u_{i},\left(\mathcal{L}\left(a_{i}^{*} a_{j}\right)-a_{i}^{*} \mathcal{L}\left(a_{j}\right)-\mathcal{L}\left(a_{i}^{*}\right) a_{j}+a_{i}^{*} \mathcal{L}(\mathbb{1}) a_{j}\right) u_{j}\right\rangle \\
& =\sum_{i, j=1}^{n}\left\langle u_{i}, \mathcal{L}\left(a_{i}^{*} a_{j}\right) u_{j}\right\rangle .
\end{aligned}
$$

This proves the lemma.

Theorem 3.14 Suppose that $\mathcal{A}=\mathcal{B}(\mathcal{H})$. A bounded linear map $\mathcal{L}$ on $\mathcal{A}$ such that $\mathcal{L}\left(a^{*}\right)=(\mathcal{L}(a))^{*}$ for every $a \in \mathcal{A}$ is conditionally completely positive if and only if there exists a completely positive map $\Phi$ on $\mathcal{A}$ and an element $G$ of $\mathcal{A}$ such that

$$
\mathcal{L}(a)=\Phi(a)+G^{*} a+a G
$$

for every $a \in \mathcal{A}$. Moreover the operator $G$ satisfies the inequality

$$
G+G^{*} \leq \mathcal{L}(\mathbb{1})
$$


Proof. Let us show first that an operator of the form (3.10) is conditionally completely positive. Fix an integer $n$ and denote by $G_{n}$ the operator $G \otimes \mathbb{1}$ in $\mathcal{A} \otimes \mathcal{M}_{n}$. For every $x \in \mathcal{A} \otimes \mathcal{M}_{n}$ we have

$$
\mathcal{L}^{(n)}(x)=\Phi^{(n)}(x)+G_{n}^{*} x+x G_{n}
$$

A straightforward computation shows that the left-hand side of (3.9) is equal to

$$
\Phi^{(n)}\left(x^{*} x\right)-\Phi^{(n)}\left(x^{*}\right) x-x^{*} \Phi^{(n)}(x)+x^{*} \Phi^{(n)}(\mathbb{1}) x .
$$

Since $\Phi$ is completely positive, then $\Phi^{(n)}$ is positive for cvery $n \geq 1$. Thus, for cvery $t \geq 0, \exp \left(t \Phi^{(n)}\right)$ is positive i.c. $\exp (t \Phi)$ is completely positive. It follows then from Proposition 3.12 that $\Phi$ is conditionally completely positive. This and the above identity imply that $\mathcal{L}$ is conditionally completely positive.

Clearly $\mathcal{L}\left(a^{*}\right)=(\mathcal{L}(a))^{*}$ because $\Phi$ is positive.

Conversely given a conditionally completely positive $\mathcal{L}$ such that $\mathcal{L}\left(a^{*}\right)=$ $(\mathcal{L}(a))^{*}$ for $a \in \mathcal{A}$ fix a unit vector $e$ in $\mathcal{H}$ and consider the operator $G$ in $\mathcal{H}$ with adjoint operator defined by

$$
G^{*} u=\mathcal{L}(|u\rangle\langle e|) e-\frac{1}{2}\langle e, \mathcal{L}(|e\rangle\langle c|) e\rangle u
$$

for $u \in \mathcal{H}$. For every $n \geq 1$ and every $a_{1}, \ldots, a_{n} \in \mathcal{A}, u_{1}, \ldots, u_{n} \in \mathcal{H}$ let

$$
u_{n+1}=e, \quad v=-\sum_{j=1}^{n} a_{j} u_{j}, \quad a_{n+1}=|v\rangle\langle e| .
$$

Clearly we have $\sum_{j=1}^{n+1} a_{j} u_{j}=0$. Applying Lemma 3.13, we obtain the inequality

$$
\begin{aligned}
0 & \leq \sum_{i, j=1}^{n}\left\langle u_{i}, \mathcal{L}\left(a_{i}^{*} a_{j}\right) u_{j}\right\rangle+\sum_{i=1}^{n}\left\langle u_{i}, \mathcal{L}\left(\left|a_{i}^{*} v\right\rangle\langle e|\right) e\right\rangle \\
& +\sum_{j=1}^{n}\left\langle e, \mathcal{L}\left(|e\rangle\left\langle a_{j}^{*} v\right|\right) u_{j}\right\rangle+\langle e, \mathcal{L}(|e\rangle\langle e|) e\rangle\|v\|^{2}
\end{aligned}
$$

By virtue of (3.11) the sum of the last three torms can be written in the form

$$
\begin{aligned}
& \sum_{i=1}^{n}\left\langle u_{i}, G^{*} a_{i}^{*} v\right\rangle+\sum_{j=1}^{n}\left\langle G^{*} a_{j}^{*} v, u_{j}\right\rangle \\
& =-\sum_{i, j=1}^{n}\left\langle u_{i}, G^{*} a_{i}^{*} a_{j} u_{j}\right\rangle-\sum_{i, j=1}^{n}\left\langle u_{i}, a_{i}^{*} a_{j} G u_{j}\right\rangle .
\end{aligned}
$$

Therefore we obtain the inequality

$$
\sum_{i, j=1}^{n}\left\langle u_{i},\left(\mathcal{L}\left(a_{i}^{*} a_{j}\right)-G^{*} a_{i}^{*} a_{j}-a_{i}^{*} a_{j} G\right) u_{j}\right\rangle \geq 0 .
$$


Proposition 2.9 then implies that the map $\Phi$ defined by

$$
\Phi(a)=\mathcal{L}(a)-G^{*} a-a G
$$

is completely positive.

Finally, the operator $G$ in (3.10) satisfies the inequality

$$
G+G^{*}=\mathcal{L}(\mathbb{1})-\Phi(\mathbb{1}) \leq \mathcal{L}(\mathbb{1}) .
$$

This completes the proof.

Remark. The choice of the operator $G$ is clearly not unique. In fact, for every $c>0$, taking $G^{\prime}=G-c \mathbb{1}$ we can write $\mathcal{L}$ in the form

$$
\mathcal{L}(a)=(\Phi+2 c \mathbb{1})(a)+G^{* *} a+a G^{\prime} .
$$

It is worth mentioning here that Christensen and Evans [30] proved Theorem 3.14 for conditionally completely positive maps on an arbitrary von Neumann algebra. However the proof is much more difficult.

We are now in a position to prove the characterisation of the infinitesimal generator of a quantum dynamical semigroup due to Lindblad [64] in the casc of an arbitrary Hilbert space $\mathcal{H}$ and to Gorini, Kossakowski and Sudarshan [55] in the case of a finite dimensional Hilbert space.

Theorem 3.15 (Lindblad) $A$ bounded operator $\mathcal{L}$ on $\mathcal{B}(\mathcal{H})$ is the infinitesimal generator of a uniformly continuous quantum dynamical semigroup if and only if there exists a complex separable Hilbert space $\mathcal{K}$, a bounded operator $L: \mathcal{H} \rightarrow$ $\mathcal{H} \otimes \mathcal{K}$ and an operator $G$ in $\mathcal{H}$ such that

$$
\mathcal{L}(a)=L^{*}(a \otimes \mathbb{1}) L+G^{*} a+a G
$$

for all $a \in \mathcal{B}(\mathcal{H})$. The operator $L$ can be chosen so that the set

$$
\{(a \otimes \mathbb{l}) L u \mid a \in \mathcal{B}(\mathcal{H}), u \in \mathcal{H}\}
$$

is total in $\mathcal{H} \otimes \mathcal{K}$.

Proof. If $\mathcal{T}$ is a quantum dynamical semigroup then the infinitesimal generator $\mathcal{L}$ is conditionally completely positive by Proposition 3.12 . Hence it can be represented in the form (3.10) by Theorem 3.14. Noreover $\mathcal{L}$ is $\sigma$-weakly continuous by Proposition 3.9. Since the map $a \rightarrow a G+G^{*} a$ is obviously $\sigma$-weakly continuous, applying Kraus' Theorem 2.20 to the map $\Phi$ we obtain the desired representation of $\mathcal{L}$.

Conversely, if the operator $\mathcal{L}$ can be represented in the form (3.12), then it is $\sigma$-weakly continuous and conditionally completely positive by Theorem 3.14. Therefore it is the infinitesimal generator of a uniformly continuous quantum dynamical semigroup by Proposition 3.9 and Proposition 3.12 . 
Remark. Notice that, when the operators $\mathcal{T}_{t}$ are not continuous in the $\sigma$-weak topology, we can obtain a similar characterization of $\mathcal{L}$ applying Stinespring's theorem instead of Kraus' theorem.

Lindblad's theorem also allows us to characterize the infinitesimal generators of contractive quantum dynamical semigroups. These can be represented in the form (3.12) with operators $G$ and $L$ satisfying the inequality

$$
\mathcal{L}(\mathbb{1})=G+G^{*}+L^{*} L \leq 0 .
$$

This remark is the starting point in the construction of quantum dynamical semigroup which are not necessarily norm continuous.

\subsection{Minimal quantum dynamical semigroup}

The class of uniformly continuous quantum dynamical semigroups is too small for the applications in quantum probability and mathematical physics (sce, for example, [10], [32], [46], [68]). The problem of constructing quantum dynamical semigroups with unbounded generator, in principle, could be treated with the Hille-Yosida theorem at least in the case when the domain of the infinitesimal generator is an algebra so that conditional complete positivity makes sense. However in all the applications the infinitesinal gonerator $\mathcal{L}$ is not given $e x$ plicitly but it is given formally in a "generalised" Lindblad form (3.12) with unbounded operators $G$ and $L$.

E.B. Davies, following Kato's paper [61], constructed in [32] the prednal semigroup of a quantum dynamical semigroup on the von Neumann algetsat $\mathcal{B}(h)$ from given operators $G$ and $L$ in $\mathcal{H}$.

A.M. Chebotarev (see [22], [23], [24j) constructed directly a quantum dynamical semigroup from the operators $G$ and $L$ in $\mathcal{H}$ generalising K.L. Chung's construction of the minimal solution of Foller-Kolmogorov equations for countable state Markov chains (see [31] Th.1 p.231 and also [49]).

Here we follow Chebotarev's construction of the minimal solution. The only assumption we make on the operators $G$ and $L$ in this section is the following:

\section{Hypothesis A}

- the operator $G$ is the infinitesimal generator of a strongly continuous contraction semigroup $\left(P_{t}\right)_{t \geq 0}$ in $h$,

- the domain of the operators $\left(L_{\ell}\right)_{\ell=1}^{\infty}$ contains the domain of $G$ and, for cvery $u \in D(G)$ we have

$$
\langle u . G u\rangle+\langle G u, u\rangle+\sum_{\ell=1}^{\infty}\left\langle L_{\ell} u, L_{\ell} u\right\rangle \leq 0 .
$$

Notice that the operators $L_{\ell}$ are not assumed to be closable. 
Lemma 3.16 Suppose that the hypothesis A holds. Then for every $\ell \geq 1$, the following conditions hold

1. the maps $t \mapsto L_{\ell} P(t) u$, are norm continuous for every $u \in D(G)$,

2. the maps $t \mapsto L_{\ell} P(t) u$ are norm differentiable for every $u \in D\left(G^{2}\right)$ and

$$
\frac{d}{d t}\left\|L_{\ell} P(t) u\right\|^{2}=L_{\ell} P(t) G u,
$$

3. the map $t \mapsto \sum_{\ell=1}^{\infty}\left\|y L_{\ell} P(t) u\right\|^{2}$ is differentiable for every $u \in D\left(G^{2}\right)$ and $y \in \mathcal{B}(h)$

$$
\frac{d}{d t} \sum_{\ell=1}^{\infty}\left\|y L_{\ell} P(t) u\right\|^{2}=2 \Re e \sum_{\ell=1}^{\infty}\left\langle y L_{\ell} P(t) u, y L_{\ell} P(t) G u\right\rangle .
$$

Proof. For each $s, t \geq 0$ and each $u \in D(G)$, the incquality (3.13) yields

$$
\sum_{\ell=1}^{\infty}\left\|L_{\ell} P(t) u-L_{\ell} P(s) u\right\|^{2} \leq-2 \mathfrak{H}_{e}\langle(P(t)-P(s)) u, G(P(t)-P(s)) u\rangle
$$

Therefore 1 follows from continuity of the map $t \rightarrow P(t) G u$.

For each $s, t$ and each $u \in D\left(G^{2}\right)$ we have

$$
\begin{aligned}
& \left\|s^{-1} L_{\ell}(P(t+s)-P(t)) u-L_{\ell} P(t) G u\right\|^{2} \\
& \quad \leq-2 \operatorname{Me}\left\langle s^{-1}(P(t+s)-P(t)) u-P(t) G u, G\left(s^{-1}(P(t+s)-P(t)) u-P(t) G u\right)\right\rangle \\
& \quad=-2 \operatorname{Me}\left\langle P(t)\left(s^{-1}(P(s)-\mathbb{1}) u-G u\right), P(t)\left(s^{-1}(P(s)-\mathbb{1}) G u-G^{2} u\right)\right\rangle .
\end{aligned}
$$

Since $u \in D\left(G^{2}\right)$ we can let $s$ tend to 0 to get the desired identity.

For each $s, t$ and each $u \in D\left(G^{2}\right)$ wo have

$$
\begin{aligned}
\sum_{\ell=1}^{\infty}\left\|y L_{\ell} P(t+s) u\right\|^{2}-\sum_{\ell=1}^{\infty}\left\|y L_{\ell} P(t) u\right\|^{2} & =\sum_{\ell=1}^{\infty}\left\|y L_{\ell} P(t+s) u-y L_{\ell} P(t) u\right\|^{2} \\
& +2 N_{\ell=1}^{\infty}\left\langle y L_{\ell} P(t) u, y L_{\ell}(P(t+s)\right. \\
& -P(t)) G u\rangle \\
& \left.=\sum_{\ell=1}^{\infty} y L_{\ell} P(t+s) u-y L_{\ell} P(t)\right) u \|^{2} \\
& +2 \Re_{\ell} \sum_{\ell=1}^{\infty}\left\langle y L_{\ell} P(t) u, y L_{\ell} \int_{t}^{t+s}(P(r)\right. \\
& -P(t)) G u d r\rangle \\
& +2 s \Re \sum_{\ell=1}^{\infty}\left\langle y L_{\ell} P(t) u, y L_{\ell} P(t) G u\right\rangle .
\end{aligned}
$$


The first term is an infinitesimum of order bigger than $s$. Indeed, by the hypothesis $\mathbf{A}$, it can be majorized by

$$
\begin{array}{r}
-2\|y\|^{2} \Re e\langle(P(t+s)-P(t)) u,(P(t+s)-P(t)) G u\rangle \\
\leq\|y\|^{2} \int_{t}^{t+s}\|P(r) G u\| d r \cdot \int_{t}^{t+s}\left\|P(r) G^{2} u\right\| d r
\end{array}
$$

The second term is also an infinitesimum of order bigger than $s$ because its modulus can be majorized by

$$
2\|y\|^{2}\left(\sum_{\ell=1}^{\infty}\left\|L_{\ell} P(t) u\right\|^{2}\right)^{1 / 2}\left(\sum_{\ell=1}^{\infty}\left\|L_{\ell} \int_{t}^{t+s}(P(r)-P(t)) G u d r\right\|^{2}\right)^{1 / 2} .
$$

The inequality (3.13) allows then to estimate the square of the only factor depending on $s$ by

$$
\begin{aligned}
-2 \Re e & \left\langle\int_{t}^{t+s}(P(r)-P(t)) G u d r, \int_{t}^{t+s}(P(r)-P(t)) G^{2} u d r\right\rangle \\
& \leq 2 \int_{t}^{t+s}\|(P(r)-P(t)) G u\| d r \cdot \int_{t}^{t+s}\left\|(P(r)-P(t)) G^{2} u\right\| d r .
\end{aligned}
$$

Therefore we have

$$
\begin{array}{r}
\lim _{s \rightarrow 0} \frac{1}{s}\left(\sum_{\ell=1}^{\infty}\left\|y L_{\ell} P(t+s) u\right\|^{2}-\sum_{\ell=1}^{\infty}\left\|y L_{\ell} P(t) u\right\|^{2}\right) \\
=2 \Re R e \sum_{\ell=1}^{\infty}\left\langle y L_{\ell} P(t) u, y L_{\ell} P(t) G u\right\rangle .
\end{array}
$$

This proves 3 .

Lemma 3.17 Let $E=\left\{(t, s) \in \mathbb{R}^{2} \mid 0 \leq s \leq t\right\}$ and let $f: E \rightarrow \mathbb{R}$ be a continuous function such that its partial derivative with respect to the first variable is also continuous. Then

$$
\frac{\partial}{\partial t} \int_{0}^{t} f(t, s) d s=f(t, t)+\int_{0}^{t} \frac{\partial f(t, s)}{\partial t} d s .
$$

The proof is elementary. We omit it.

We shall denote by $\|\cdot\|_{\infty}$ the norm in $\mathcal{B}(h)$.

For all $x \in \mathcal{B}(h)$ consider the sesquilinear form $£(x)$ in $h$ with domain $D(G) \times D(G)$ given by

$$
\langle v, £(x) u\rangle=\langle v, x G u\rangle+\langle G v, x u\rangle+\sum_{\ell=1}^{\infty}\left\langle L_{\ell} v, x L_{\ell} u\right\rangle
$$

We want to construct a quantum dynamical semigroup satisfying the equation

$$
\left\langle v, \mathcal{T}_{t}(x) u\right\rangle=\langle v, x u\rangle+\int_{0}^{\iota}\left\langle v, £\left(\mathcal{T}_{s}(x)\right) u\right\rangle d s
$$


Proposition 3.18 Suppose that the hypothesis A holds and, for all $x \in \mathcal{B}(h)$, let $\left(\mathcal{T}_{t}(x)\right)_{t \geq 0}$ be a $\sigma$-weakly continuous family of elements of $\mathcal{B}(h)$ such that

$$
\left\|\mathcal{T}_{t}(x)\right\|_{\infty} \leq\|x\|_{\infty} .
$$

The following conditions are equivalent:

1. equation (3.15) holds for all $v, u \in D(G)$,

2. for all $v, u \in D(G)$ we have

$$
\begin{aligned}
\left\langle v, \mathcal{T}_{t}(x) u\right\rangle & =\langle P(t) v, x P(t) u\rangle \\
& +\sum_{\ell=1}^{\infty} \int_{0}^{t}\left\langle L_{\ell} P(t-s) v \cdot \mathcal{T}_{s}(x) L_{\ell} P(t-s) u\right\rangle d s
\end{aligned}
$$

Proof. We remind first that $D\left(G^{2}\right)$ is a core for $G$.

In order to show that 1 implies 2 we fix $t$ and compute the derivative

$$
\frac{d}{d s}\left\langle P(t-s) v, \mathcal{T}_{s}(x) P(t-s) u\right\rangle=\sum_{\ell=1}^{\infty}\left\langle L_{\ell} I^{\prime}(t-s) v, \mathcal{T}_{s}(x) L_{\ell} P(t-s) u\right\rangle
$$

using equation (3.15). Clcarly (3.16) follows integrating the above identity on the interval $[0, t]$.

We prove now that condition 2 inplies condition 1. For each $v, u \in D\left(G^{2}\right)$. by using Lemma 3.16 3, Lenma 3.17 and the complex polarisation identity. we compute the derivative

$$
\begin{aligned}
\frac{d}{d t}\left\langle v, \mathcal{T}_{t}(x) u\right\rangle & =\langle P(t) v, x P(t) G u\rangle+\langle P(t) G v, x P(t) u\rangle+\sum_{\ell=1}^{\infty}\left\langle L_{\ell} v, \mathcal{T}_{t}(x) L_{\ell} u\right\rangle \\
& +\int_{0}^{t} \sum_{\ell=1}^{\infty}\left\langle L_{\ell} P(t-s) v, \mathcal{T}_{s}(x) L_{\ell} P(t-s) G u\right\rangle d s \\
& +\int_{0}^{\ell} \sum_{\ell=1}^{\infty}\left\langle L_{\ell} P(t-s) G v \cdot \mathcal{T}_{s}(x) L_{\ell} P(t-s) u\right\rangle d s .
\end{aligned}
$$

The sum of the first and fourth (resp. second and fifth) term equals

$$
\left\langle v, \mathcal{T}_{t}(x) G u\right\rangle, \quad\left(\text { resp. }\left\langle G v, \mathcal{T}_{t}(x) u\right\rangle\right)
$$

because of (3.16). The right-hand side of the above equation thus coincides with $\left\langle v, £\left(\mathcal{T}_{t}(x)\right) u\right\rangle$. Therefore (3.15), for $v, u \in D\left(G^{2}\right)$, follows by integrating on $[0, t]$. Since $D\left(G^{2}\right)$ is a core for $G$ the proof is complete.

We construct now a solution of (3.16) by iteration. 
Proposition 3.19 Suppose that the hypothesis A holds. Then there exists a sequence $\left(\mathcal{T}_{t}^{(n)}\right)_{n \geq 0}$ of linear contractions on $\mathcal{B}(h)$ which satisfy

$$
\begin{aligned}
\left\langle v, \mathcal{T}_{t}^{(0)}(x) u\right\rangle & =\langle P(t) v, x P(t) u\rangle \\
\left\langle v, \mathcal{T}_{t}^{(n+1)}(x) u\right\rangle & =\langle P(t) v, x P(t) u\rangle \\
& +\sum_{\ell=1}^{\infty} \int_{0}^{t}\left\langle L_{\ell} P(t-s) v, \mathcal{T}_{s}^{(n)}(x) L_{\ell} P(t-s) u\right\rangle d s
\end{aligned}
$$

for $t \geq 0, x \in \mathcal{B}(h), u, v \in D(G)$. Furthermore:

1. the map $\mathcal{T}_{t}^{(n)}: \mathcal{B}(h) \rightarrow \mathcal{B}(h)$ is completely positive for every $t \geq 0$ and severy $n \geq 0$,

$\therefore$ the map $\mathcal{T}_{t}^{(n)}: \mathcal{B}(h) \rightarrow \mathcal{B}(h)$ is normal for every $t \geq 0$ and every $n \geq 0$,

3. the map $t \rightarrow \mathcal{T}_{t}^{(n)}(x)$ is $\sigma$-weakly continuous for every $x \in \mathcal{B}(h)$,

4. the sequence $\left(\mathcal{T}_{t}^{(n)}(x)\right)_{n \geq 0}$ is increasing for every positive $x \in \mathcal{B}(h)$,

5. $\mathcal{T}_{t}^{(n)}(\mathbb{1}) \leq \mathbb{1}$ for every $n \geq 0, t \geq 0$.

Proof. Clearly the linear maps $\mathcal{T}_{t}^{(0)}$ are well defined and enjoy all the above properties (see Example 3.1).

Suppose that the linear maps $\mathcal{T}_{t}^{(n)}$ are contractions on $\mathcal{B}(h)$ satisfying 12 and 3 for a fixed integer $n$. Let us establish that the maps $\mathcal{T}_{t}^{(n+1)}$ enjoy the same properties.

For every $x \in \mathcal{B}(h)$ and $v, u \in D(G)$ the integral in the right hand side of (3.17) is well defined because of the properties of maps $\mathcal{T}_{t}^{(n)}$. Moreover, by using the Schwarz inequality, we have

$$
\begin{aligned}
& \left|\sum_{\ell=1}^{\infty} \int_{0}^{t}\left\langle L_{\ell} P(t-s) v, \mathcal{T}_{s}^{(n)}(x) L_{\ell} P(t-s) u\right\rangle d s\right| \\
\leq & \|x\|_{\infty} \sum_{\ell=1}^{\infty} \int_{0}^{t}\left\|L_{\ell} P(t-s) v^{\prime}\right\|\left\|L_{\ell} P(t-s) u\right\| d s \\
\leq & \|x\|_{\infty}\left(\sum_{\ell=1}^{\infty} \int_{0}^{t}\left\|L_{\ell} P(t-s) v\right\|^{2} d s\right)^{\frac{1}{2}}\left(\sum_{\ell=1}^{\infty} \int_{0}^{t}\left\|L_{\ell} P(t-s) u\right\|^{2} d s\right)^{\frac{1}{2}} .
\end{aligned}
$$

By virtue of the inequality (3.13) in hypothesis $\mathbf{A}$, the square of the third factor in the right-hand side can be estimated by the square root of

$$
\begin{aligned}
\int_{0}^{t}-2 \Re e\langle P(t-s) u, G P(t-s) u\rangle d s & =\int_{0}^{t} \frac{d}{d t}\|P(t-s)\|^{2} d s \\
& =\|u\|^{2}-\|P(t) u\|^{2}
\end{aligned}
$$


Hence, due to the elementary inequality

$$
\left(a^{2}-b^{2}\right)^{1 / 2}\left(c^{2}-d^{2}\right)^{1 / 2} \leq a c-b d,
$$

for every non negative real numbers such that $a \geq b$ and $c \geq d$ we have the estimate

$$
\begin{aligned}
\left|\sum_{\ell=1}^{\infty} \int_{0}^{t}\left\langle L_{\ell} P(t-s) v, \mathcal{T}_{s}^{(n)}(x) L_{\ell} P(t-s) u\right\rangle d s\right| \\
\leq\|x\|_{\infty}(\|v\| \cdot\|u\|-\|P(t) v\| \cdot\|P(t) u\|)
\end{aligned}
$$

Since the scalar product $\langle P(t) v, x P(t) u\rangle$ can be obviously estimated by $\|x\|_{\infty}$. $\|P(t) v\| \cdot\|P(t) u\|$, the right-hand side of (3.17) is majorized by

$$
\|x\|_{\infty} \cdot\|v\| \cdot\|u\| .
$$

Therefore it defines a contractive operator $\mathcal{T}_{t}^{(n+1)}$ in $\mathcal{B}(h)$.

The operator $\mathcal{T}_{t}^{(n+1)}$ is completely positive. In fact, for every integer $m \geq 1$, vectors $u_{1}, \ldots, u_{m} \in D(G)$ and operators $x_{1}, \ldots, x_{n} \in \mathcal{B}(h)$ we have

$$
\begin{aligned}
& \sum_{i, j=1}^{m}\left\langle u_{i}, \mathcal{T}_{t}^{(n+1)}\left(x_{i}^{*} x_{j}\right) u_{j}\right\rangle=\sum_{i, j=1}^{m}\left\langle P(t) u_{i}, x_{i}^{*} x_{j} P(t) u_{j}\right\rangle \\
+ & \sum_{i, j=1}^{m} \sum_{\ell=1}^{\infty} \int_{0}^{t}\left\langle L_{\ell} P(t-s) u_{i}, \mathcal{T}_{s}^{(n)}\left(x_{i}^{*} x_{j}\right) L_{\ell} P(t-s) u_{j}\right\rangle d s .
\end{aligned}
$$

The right-hand side is positive due to the induction hypothesis. Therefore the operator $\mathcal{T}_{t}^{(n+1)}$ is completely positive by Proposition 2.9 .

We prove now, again by induction, that the property 2 . Clearly 2 holds for $n=0$. Suppose that it has been established for an integer $n$. In order to prove it for the integer $n+1$ it suffices to show that, for every $u \in D(G)$ and every increasing net $\left(x_{\alpha}\right)$ of positive elements of $\mathcal{B}(h)$ converging to an element $x$ of $\mathcal{B}(h)$ the supremum over $\alpha$ of

$$
\sum_{\ell=1}^{\infty} \int_{0}^{t}\left\langle L_{\ell} P(t-s) u, \mathcal{T}_{s}^{(n)}\left(x_{\alpha}\right) L_{\ell} P(t-s) u\right\rangle d s
$$

coincides with

$$
\sum_{\ell=1}^{\infty} \int_{0}^{t}\left\langle L_{\ell} P(t-s) u, \mathcal{T}_{s}^{(n)}(x) L_{\ell} P(t-s) u\right\rangle d s .
$$

Since all the terms in the above sums are positive and the series is convergent we can exchange the supremum and summation over $\ell$. Thus it suffices to show the above property for each term of the sum.

The family of positive continuous functions on $[0, t]$ (with parameter $\alpha$ )

$$
s \rightarrow\left\langle L_{\ell} P(t-s) u, \mathcal{T}_{s}^{(n)}\left(x_{\alpha}\right) L_{\ell} P(t-s) u\right\rangle
$$


is increasing and converges pointwise to the continuous function

$$
s \rightarrow\left\langle L_{\ell} P(t-s) u, \mathcal{T}_{s}^{(n)}(x) L_{\ell} P(t-s) u\right\rangle .
$$

Dini's lemma implies then that it converges uniformly. Thus we can exchange the supremum over $\alpha$ and the integral on $[0, t]$ to obtain the desired property for the integer $n+1$.

In a similar way, using the induction hypothesis, it is easy to see that the operators $\mathcal{T}_{t}^{(n+1)}$ enjoy properties the property 3 .

Property 4 follows by induction from the identity

$$
\begin{aligned}
& \left\langle u,\left(\mathcal{T}_{t}^{(n+1)}(x)-\mathcal{T}_{i}^{(n)}(x)\right) u\right\rangle \\
= & \sum_{\ell=1}^{\infty} \int_{0}^{t}\left\langle L_{\ell} P(t-s) u,\left(\mathcal{T}_{s}^{(n)}(x)-\mathcal{T}_{s}^{(n-1)}(x)\right) L_{\ell} P(t-s) u\right\rangle d s
\end{aligned}
$$

for every $n \geq 1$.

Finally, notice that the operators $\mathcal{T}_{t}$ are contractive so that $\left\|\mathcal{T}_{i}(\mathbb{1})\right\| \leq 1$. Thus, since they are also positive, we have $0 \leq \mathcal{T}_{t}(\mathbb{1}) \leq \mathbb{1}$ This completes the proof.

Lemma 3.20 Suppose that the hypothesis A holds. Then there exists a family $\left(\mathcal{T}_{t}\right)_{\geq 0}$ of contractive linear maps on $\mathcal{B}(h)$ such that:

1. $\mathcal{T}_{t}$ is completely positive for cvery $t \geq 0$,

2. $\mathcal{T}_{t}$ is normal for every $t \geq 0$,

3. the family $\left(\mathcal{T}_{t}(x)\right)_{t \geq 0}$ of linear operators on $\mathcal{B}(h)$ solves equations (3.15) and (3.16) for all $x \in \mathcal{B}(h)$,

4. for every $x \in \mathcal{B}(h)$ the map $t \rightarrow \mathcal{T}_{t}(x)$ is continuous with respect to the $\sigma$-weak topology on $\mathcal{B}(h)$.

Proof. Fix $t \geq 0$ and let $\left(\mathcal{T}_{t}^{(n)}\right)_{n \geq 0}$ be the sequence of positive lincar operators on $\mathcal{B}(h)$ constructed in Proposition 3.19.

For every positive operator $x \in \mathcal{B}(h)$ and every $t \geq 0$ the sequence $\left(\mathcal{T}_{t}^{(n)}(x)\right)_{n \geq 0}$ is increasing and bounded from above by $\|x\|_{\infty} \mathbb{1}$. Therefore the limit

$$
\lim _{n \rightarrow \infty}\left\langle u, \mathcal{T}_{t}^{(n)}(x) u\right\rangle
$$

exists for every $u \in h$.

By the complex polarisation formula,

$$
4\left\langle v, \mathcal{T}_{t}(x) u\right\rangle=\sum_{k=0}^{3} i^{-k}\left\langle v+i^{k} u, \mathcal{T}_{t}(x)\left(v+i^{k} u\right)\right\rangle
$$


it follows that the limit

$$
\lim _{n \rightarrow \infty}\left\langle v, \mathcal{T}_{t}^{(n)}(x) u\right\rangle
$$

exists for every $v, u \in h$.

Writing an arbitrary element $x$ of $\mathcal{B}(h)$ first as the sum

$$
x=\frac{x+x^{*}}{2}+i \frac{x-x^{*}}{2 i}
$$

of two self-adjoint operators in $\mathcal{B}(h)$ and then writing each one of these selfadjoint operators as the difference of their positive and negative part we can easily see that the limit (3.19) exists for every $v, u \in h$ and every $x \in \mathcal{B}(h)$. Moreover, since the operators $\mathcal{T}_{t}^{(n)}$ are contractions, we have

$$
\left|\lim _{n \rightarrow \infty}\left\langle v, \mathcal{T}_{t}^{(n)}(x) u\right\rangle\right| \leq\|x\|_{\infty} \cdot\|v\| \cdot\|u\| .
$$

It follows that there exist contractions $\mathcal{T}_{t}$ in $\mathcal{B}(h)$ such that

$$
\left\langle v, \mathcal{T}_{t}(x) u\right\rangle=\lim _{n \rightarrow \infty}\left\langle v, \mathcal{T}_{t}^{(n)}(x) u\right\rangle .
$$

Clearly the maps $\mathcal{T}_{t}$ and property 1 then follows from Proposition 2.15.

In order to prove 2 consider an increasing net $\left(x_{\alpha}\right)$ of positive operators in $\mathcal{B}(h)$ with least upper bound $x$. Since the maps $\mathcal{T}_{t}^{(n)}(n \geq 0)$ are $\sigma$-weakly continuous, for every $u \in h$ we have

$$
\begin{aligned}
\sup _{\alpha}\left\langle u, \mathcal{T}_{t}\left(x_{\alpha}\right) u\right\rangle & =\sup _{\alpha} \sup _{n}\left\langle u, \mathcal{T}_{t}^{(n)}\left(x_{\alpha}\right) u\right\rangle \\
& =\sup _{n} \sup _{\alpha}\left\langle u, \mathcal{T}_{t}^{(n)}\left(x_{\alpha}\right) u\right\rangle \\
& =\sup _{n}\left\langle u, \mathcal{T}_{t}^{(n)}(x) u\right\rangle \\
& =\left\langle u, \mathcal{T}_{t}(x) u\right\rangle .
\end{aligned}
$$

This shows that the maps $\mathcal{T}_{t}$ are normal.

Letting $n$ tend to infinity in (3.17) it follows that equation (3.16) holds. Equation (3.15) also holds because of Proposition 3.18.

Finally, for every $u, v \in h$ and $x \in \mathcal{B}(h)$, the map

$$
t \rightarrow\left\langle v, \mathcal{T}_{t}(x) u\right\rangle
$$

is continuous because of equation (3.16). Therefore the uniformly bounded map $t \rightarrow \mathcal{T}_{t}(x)$ is continuous with respect to the weak and $\sigma$-weak topology on $\mathcal{B}(h)$, completing the proof.

The following Lemma, which can be considered as a quantum version of a classical formula (see [31] Ch.2, Sect. 18, p. 230), is a fundamental tool in the proof of the semigroup property for the operators $\left(\mathcal{T}_{t}\right)_{t \geq 0}$ constructed in Lemma 3.20 . 
Lemma 3.21 Suppose that the hypothesis $\mathbf{A}$ holds. Let $\left(\mathcal{W}_{t}^{(n)}\right)_{n \geq 0}$ be the sequence of positive linear maps on $\mathcal{B}(h)$ defined inductively by

$$
\begin{aligned}
\mathcal{W}_{t}^{(0)} & =\mathcal{T}_{t}^{(0)} \\
\mathcal{W}_{t}^{(n+1)} & =\mathcal{T}_{t}^{(n+1)}-\mathcal{T}_{t}^{(n)} .
\end{aligned}
$$

Then, for each $n \geq 0$, the following identity holds

$$
\mathcal{W}_{t+s}^{(n)}(x)=\sum_{k=0}^{n} \mathcal{W}_{t}^{(k)}\left(\mathcal{W}_{s}^{(n-k)}(x)\right)
$$

Proof. Clearly from (3.17), we have

$$
\left\langle u, \mathcal{W}_{t}^{(n+1)}(x) u\right\rangle=\sum_{\ell=1}^{\infty} \int_{0}^{t}\left\langle L_{\ell} P(t-s) v, \mathcal{W}_{s}^{(n)}(x) L_{\ell} P(t-s) u\right\rangle d s
$$

for $v, u \in D(G), x \in \mathcal{B}(h)$ and $n \geq 0$.

Identity (3.21) clearly holds for $n=0$. Assume that it has been established for a given $n$, then, using (3.22), for every $v, u \in D(G), x \in \mathcal{B}(h)$, we have

$$
\begin{aligned}
& \sum_{k=0}^{n+1}\left\langle v, \mathcal{W}_{t}^{(k)}\left(\mathcal{W}_{s}^{(n+1-k)}(x)\right) u\right\rangle \\
= & \left\langle v, \mathcal{W}_{t}^{(0)}\left(\mathcal{W}_{s}^{(n+1)}(x)\right) u\right\rangle \\
+ & \sum_{k=0}^{n} \sum_{\ell=1}^{\infty} \int_{0}^{t}\left\langle L_{\ell} P(t-r) v, \mathcal{W}_{r}^{(k)}\left(\mathcal{W}_{s}^{(n-k)}(x)\right) L_{\ell} P(t-r) u\right\rangle d r
\end{aligned}
$$

Identity (3.21) for the given $n$ and the semigroup property of $(P(t))_{t \geq 0}$ yield then

$$
\begin{aligned}
& \sum_{k=0}^{n+1}\left\langle v, \mathcal{W}_{t}^{(k)}\left(\mathcal{W}_{s}^{(n+1-k)}(x)\right) u\right\rangle \\
= & \left\langle v, \mathcal{W}_{t}^{(0)}\left(\mathcal{W}_{s}^{(n+1)}(x)\right) u\right\rangle \\
+ & \sum_{\ell=1}^{\infty} \int_{0}^{t}\left\langle L_{\ell} P(t-r) v, \mathcal{W}_{s+r}^{(n)}(x) L_{\ell} P(t-r) u\right\rangle d r \\
= & \left\langle P(t) v, \mathcal{W}_{s}^{(n+1)}(x) P(t) u\right\rangle \\
+ & \sum_{\ell=1}^{\infty} \int_{s}^{t+s}\left\langle L_{\ell} P(t+s-r) v, \mathcal{W}_{r}^{(n)}(x) L_{\ell} P(t+s-r) u\right\rangle d r \\
= & \left\langle P(t) v, \mathcal{W}_{s}^{(n+1)}(x) P(t) u\right\rangle \\
- & \sum_{\ell=1}^{\infty} \int_{0}^{s}\left\langle L_{\ell} P(s-r) P(t) v, \mathcal{W}_{r}^{(n)}(x) L_{\ell} P(s-r) P(t) u\right\rangle d r
\end{aligned}
$$




$$
+\sum_{\ell=1}^{\infty} \int_{0}^{t+s}\left\langle L_{\ell} P(t+s-r) v, \mathcal{W}_{r}^{(n)}(x) L_{\ell} P(t+s-r) u\right\rangle d r
$$

The sum of the first two terms vanishes and the third one is equal to the scalar product $\left\langle v, \mathcal{W}_{t+s}^{(n)}(x) u\right\rangle$ by virtue of (3.22). This proves identity (3.21).

Theorem 3.22 Suppose that the hypothesis $\mathbf{A}$ holds. Then there exists a quantum dynamical semigroup $\left(\mathcal{T}_{t}^{(\mathrm{min})}\right)_{t \geq 0}$ solving equations (3.16), (3.15) with the following properties:

1. $\mathcal{T}_{t}^{(\min )}(\mathbb{1}) \leq \mathbb{1}$ for every $t \geq 0$,

2. for every $\sigma$-weakly continuous family $\left(\mathcal{T}_{t}\right)_{t \geq 0}$ of positive maps on $\mathcal{B}(h)$ satisfying (3.16) and (3.15) and every positive operator $x \in \mathcal{B}(h)$, we have

$$
\mathcal{T}_{t}^{(\min )}(x) \leq \mathcal{T}_{l}(x)
$$

for all $t \geq 0$.

Proof. Let $\left(\mathcal{T}_{t}^{(\min )}\right)_{t \geq 0}$ be the family of linear maps constructed in Lemma 3.20. In order to show that it is a quantum dynamical semigroup clearly it suffices to prove the semigroup property.

$$
\mathcal{T}_{t+s}^{(\min )}(x)=\mathcal{T}_{t}^{(\min )}\left(\mathcal{T}_{s}^{(\min )}(x)\right)
$$

for $x \in \mathcal{B}(h)$ and $t, s \geq 0$. By Lemma 3.21 we have the identities

$$
\begin{aligned}
\left\langle u, \mathcal{T}_{t+s}^{(n)}(x) u\right\rangle & =\sum_{k=0}^{n}\left\langle u, \mathcal{W}_{t+s}^{(k)}(x) u\right\rangle \\
& =\sum_{k=0}^{n} \sum_{j=0}^{k}\left\langle u, \mathcal{W}_{t}^{(j)}\left(\mathcal{W}_{s}^{(k-j)}(x)\right) u\right\rangle
\end{aligned}
$$

for every $u \in h$ and every positive operator $x$ in $\mathcal{B}(h)$. Exchanging summations over $k$ and $j$ we obtain

$$
\left\langle u, \mathcal{T}_{t+s}^{(n)}(x) u\right\rangle=\sum_{j=0}^{n}\left\langle u \cdot \mathcal{W}_{t}^{(j)}\left(\mathcal{T}_{s}^{(n-j)}(x)\right) u\right\rangle .
$$

Since the maps $\mathcal{W}_{t}^{(j)}$ are $\sigma$-weakly continuous, we can let $n$ tend to infinity to get

$$
\begin{aligned}
\left\langle u, \mathcal{T}_{t+s}^{(\min )}(x) u\right\rangle & =\sum_{j=0}^{\infty}\left\langle u, \mathcal{W}_{t}^{(j)}\left(\mathcal{T}_{s}^{(\min )}(x)\right) u\right\rangle \\
& =\left\langle u, \mathcal{T}_{t}^{(\min )}\left(\mathcal{T}_{s}^{(\min )}(x)\right) u\right\rangle .
\end{aligned}
$$


By complex polarization we have also

$$
\left\langle v, \mathcal{T}_{t+s}^{(\min )}(x) u\right\rangle=\left\langle v, \mathcal{T}_{t}^{(\min )}\left(\mathcal{T}_{s}^{(\min )}(x)\right) u\right\rangle
$$

for every $v, u \in h$. Therefore, writing an arbitrary $x \in \mathcal{B}(h)$ as a linear combination of positive self-adjoint operators in $\mathcal{B}(h)$ we obtain the semigroup law (3.24).

This proves that $\mathcal{T}^{(\min )}$ is a quantum dynamical semigroup. Property 1 . clearly follows from condition 5 in Proposition 3.19.

Finally, for every $\sigma$-weakly continuous family $\left(\mathcal{T}_{t}\right)_{t \geq 0}$ of positive maps on $\mathcal{B}(h)$ satisfying (3.16) and (3.15) and every positive operator $x \in \mathcal{B}(h)$, we have

$$
\mathcal{T}_{t}(x) \geq P(t)^{*} x P(t)=\mathcal{T}_{t}^{(0)}(x)
$$

and. for $n \geq 0, u \in h$,

$$
\begin{aligned}
& \left\langle u,\left(\mathcal{T}_{t}(x)-\mathcal{T}_{t}^{(n+1)}(x)\right) u\right\rangle \\
= & \sum_{\ell=1}^{\infty} \int_{0}^{t}\left\langle L_{\ell} P(t-s) u \cdot\left(\mathcal{T}_{s}(x)-\mathcal{T}_{s}^{(n)}(x)\right) L_{\ell} P(t-s) u\right\rangle d s .
\end{aligned}
$$

Therefore a straightforward induction argument yields the inequality

$$
\mathcal{T}_{t}^{(n)}(x) \leq \mathcal{T}_{t}(x)
$$

for every $n \geq 0$. This implies (3.23) by construction of $\mathcal{T}^{\text {(min) }}$.

The following corollary (see [40] Corollary 4.4 p. 159, [19] Remark 2.1 p.91) gives a simple and useful property of Markov minimal quant um dynamical semigroups.

Corollary 3.23 Suppose that the hypothesis A holds and that the minimal quantum dynamical semigroup $\mathcal{T}^{(\mathrm{min})}$ is Markov. Then it is the unique $\sigma$-weakly continuous family $\left(\mathcal{T}_{t}\right)_{l \geq 0}$ of positive maps on $\mathcal{B}(h)$ satisfying (3.15).

Proof. Let $\mathcal{T}$ be $\sigma$-weakly continuous family of positive maps satisfying (3.15). Applying Theorem 3.22, for every $x \in \mathcal{B}(h)$ such that $0 \leq x \leq \mathbb{1}$ and every $t \geq 0$, we have

$$
\begin{aligned}
\mathcal{T}_{t}^{(\min )}(x) & \leq \mathcal{T}_{t}(x) \\
& =\mathcal{T}_{t}(\mathbb{1})-\mathcal{T}_{t}(\mathbb{1}-x) \\
& \leq \mathbb{1}-\mathcal{T}_{t}(\mathbb{1}-x) \\
& \leq \mathbb{1}-\mathcal{T}_{t}^{(\min )}(\mathbb{1}-x) \\
& =\mathcal{T}_{t}^{(\min )}(x) .
\end{aligned}
$$

Since every $y \in \mathcal{B}(h)$ can be written as a linear combination of four such operators $x$, it follows that $\mathcal{T}_{t}^{(\min )}(y)=\mathcal{T}_{t}(y)$ for every $y \in \mathcal{B}(h)$. 


\subsection{The resolvent of the minimal semigroup}

In the previous section we constructed the minimal quantum dynamical semigroup associated with operators $G, L_{\ell}$ satisfying the hypothesis $\mathbf{A}$. This semigroup satisfies the equations (3.15) and (3.16) but, in general, it is not characterised by this property. Moreover we would like identify the domain of the infinitesimal generator of $\mathcal{T}^{(\mathrm{min})}$ knowing only the operators $G, L_{\ell}$.

As a first step in the study of these problems we give a useful explicit representation of the resolvent of the minimal quantum dynamical semigroup obtained by A.M. Chebotarev (see [25], Lemma 4).

We follow the article [28] Sect. 3 (see also [26] Sect. 2)

Proposition 3.24 Suppose that the hypothesis A holds. The linear positive maps $\mathcal{P}_{\lambda}: \mathcal{B}(h) \rightarrow \mathcal{B}(h)$ and $\mathcal{Q}_{\lambda}: \mathcal{B}(h) \rightarrow \mathcal{B}(h)$ defined by

$$
\begin{aligned}
\left\langle v, \mathcal{P}_{\lambda}(x) u\right\rangle & =\int_{0}^{\infty} \exp (-\lambda s)\langle P(s) v, x P(s) u\rangle d s \\
\left\langle v, \mathcal{Q}_{\lambda}(x) u\right\rangle & =\sum_{\ell=1}^{\infty} \int_{0}^{\infty} \exp (-\lambda s)\left\langle L_{\ell} P(s) v, x L_{\ell} P(s) u\right\rangle d s
\end{aligned}
$$

for $\lambda>0$ and $x \in \mathcal{B}(h), v, u \in D(G)$ are normal and completely positive. Moreover we have

$$
\left\|\mathcal{P}_{\lambda}\right\|_{\infty} \leq \lambda^{-1}, \quad\left\|\mathcal{Q}_{\lambda}\right\|_{\infty} \leq 1 .
$$

Proof. The map $\mathcal{P}_{\lambda}$ is well defined, normal and completely positive by Proposition 3.3. Moreover, for every $u, v \in h$ and $x \in \mathcal{B}(h)$, we have the estimates

$$
\begin{aligned}
\left|\left\langle v, \mathcal{P}_{\lambda}(x) u\right\rangle\right| & \leq\|x\|_{\infty} \int_{0}^{\infty} \exp (-\lambda s)\|P(s) v\| \cdot\|P(s) u\| d s \\
& \leq\|x\|_{\infty}\left(\int_{0}^{\infty} \mathrm{e}^{-\lambda s}\|v\|^{2} d s\right)^{1 / 2} \cdot\left(\int_{0}^{\infty} \mathrm{e}^{-\lambda s}\|u\|^{2} d s\right)^{1 / 2} \\
& =\lambda^{-1}\|x\|_{\infty} \cdot\|v\| \cdot\|u\| .
\end{aligned}
$$

The linear map $\mathcal{Q}_{\lambda}$ is also well defined and contractive. In fact the modulus of the right-hand side of (3.25) can be estimated by $\|x\|_{\infty}$ times

$$
\begin{aligned}
& \sum_{\ell=1}^{\infty} \int_{0}^{\infty} \mathrm{e}^{-\lambda s}\left\|L_{\ell} P(s) v\right\| \cdot\left\|L_{\ell} P(s) u\right\| d s \\
\leq & \left(\sum_{\ell=1}^{\infty} \int_{0}^{\infty} \mathrm{e}^{-\lambda s}\left\|L_{\ell} P(s) v\right\|^{2} d s\right)^{1 / 2} \cdot\left(\sum_{\ell=1}^{\infty} \int_{0}^{\infty} \mathrm{e}^{-\lambda s}\left\|L_{\ell} P(s) u\right\|^{2} d s\right)^{1 / 2} .
\end{aligned}
$$

Estimating each integral as in (3.18) we obtain

$$
\left|\left\langle v, \mathcal{Q}_{\lambda}(x) u\right\rangle\right| \leq\|x\|_{\infty} \cdot\|v\| \cdot\|u\| .
$$


This proves that $\mathcal{Q}_{\lambda}$ is well defined and contractive. The argument of the proof of Proposition 3.19 shows that it is also normal and completely positive.

The resolvent $\left(\mathcal{R}_{\lambda}^{(\mathrm{min})}\right)_{\lambda>0}$ of the minimal quantum dynamical semigroup, which is characterised by the equation

$$
\left\langle v, \mathcal{R}_{\lambda}^{(\min )}(x) u\right\rangle=\int_{0}^{\infty} \exp (-\lambda s)\left\langle v, \mathcal{T}_{s}^{(\min )}(x) u\right\rangle d s
$$

with $x \in \mathcal{B}(h)$ and $v, u \in h$, admits the following representation (see [28] Th. 3.1):

Theorem 3.25 For every $\lambda>0$ and $x \in \mathcal{B}(h)$ we have

$$
\mathcal{R}_{\lambda}^{(\min )}(x)=\sum_{k=0}^{\infty} \mathcal{Q}_{\lambda}^{k}\left(\mathcal{P}_{\lambda}(x)\right)
$$

the series being convergent for the strong operator topology.

Proof. Let $\left(\mathcal{R}_{\lambda}^{(n)}\right)_{n \geq 0}$ be the sequence of linear monotone maps $\mathcal{R}_{\lambda}^{(n)}: \mathcal{B}(h) \rightarrow$ $\mathcal{B}(h)$ given by

$$
\left\langle v, \mathcal{R}_{\lambda}^{(n)}(x) u\right\rangle=\int_{0}^{\infty} \exp (-\lambda s)\left\langle v, \mathcal{T}_{s}^{(n)}(x) u\right\rangle d s
$$

where the maps $\mathcal{T}_{s}^{(n)}$ are defined by (3.17). Since the maps $\mathcal{T}_{s}^{(n)}$ are contractions $\mathcal{R}_{\lambda}^{(n)}$ is well defined. Moreover, for all positive element $x$ of $\mathcal{B}(h)$, the sequence $\left(\mathcal{R}_{\lambda}^{(n)}(x)\right)_{n \geq 0}$ is non-decreasing. Therefore, by the definition of minimal quantum dynamical semigroup, for all $u \in h$ we have

$$
\sup _{n \geq 0}\left\langle u, \mathcal{R}_{\lambda}^{(n)}(x) u\right\rangle=\left\langle u, \mathcal{R}_{\lambda}^{(\min )}(x) u\right\rangle=\int_{0}^{\infty} \exp (-\lambda s)\left\langle u, \mathcal{T}_{s}^{(\min )}(x) u\right\rangle d s .
$$

Equation (3.17) yields

$$
\begin{gathered}
\left\langle u, \mathcal{R}_{\lambda}^{(n+1)}(x) u\right\rangle=\int_{0}^{\infty} \mathrm{e}^{-\lambda t}\langle P(t) v, x P(t) u\rangle d t \\
+\sum_{\ell=1}^{\infty} \int_{0}^{\infty} \mathrm{e}^{-\lambda t} d t \int_{0}^{t}\left\langle L_{\ell} P(t-s) u, \mathcal{T}_{s}^{(n)}(x) L_{\ell} P(t-s) u\right\rangle d s
\end{gathered}
$$

for all $u, v \in D(G)$. By the change of variables $(r, s)=(t-s, s)$ in the above double integral we have

$$
\begin{aligned}
& \left\langle u, \mathcal{R}_{\lambda}^{(n+1)}(x) u\right\rangle=\left\langle u, \mathcal{P}_{\lambda}(x) u\right\rangle \\
& \quad+\sum_{\ell=1}^{\infty} \int_{0}^{\infty} \mathrm{e}^{-\lambda r} d r \int_{0}^{\infty} \mathrm{e}^{-\lambda s}\left\langle L_{\ell} P(r) u, \mathcal{T}_{s}^{(n)}(x) L_{\ell} P(r) u\right\rangle d s \\
& \quad=\left\langle u, \mathcal{P}_{\lambda}(x) u\right\rangle+\sum_{\ell \geq 1} \int_{0}^{\infty} \mathrm{e}^{-\lambda r}\left\langle L_{\ell} P(r) u, \mathcal{R}_{\lambda}^{(n)}(x) L_{\ell} P(r) u\right\rangle d r
\end{aligned}
$$


Therefore we obtain the recursion formula

$$
\mathcal{R}_{\lambda}^{(n+1)}(x)=\mathcal{P}_{\lambda}(x)+\mathcal{Q}_{\lambda}\left(\mathcal{R}_{\lambda}^{(n)}(x)\right)
$$

Iterating $n$ times this equation we have

$$
\mathcal{R}_{\lambda}^{(n+1)}(x)=\sum_{k=0}^{n+1} \mathcal{Q}_{\lambda}^{k}\left(\mathcal{P}_{\lambda}(x)\right)
$$

and (3.26) follows letting $n$ tend to $+\infty$. Formula (3.26) also holds for an arbitrary element of $\mathcal{B}(h)$ since each bounded operator can be written as a linear combination of positive self-adjoint operators.

The domain of the infinitesimal generator of the minimal quantum dynamical semigroup $\mathcal{T}^{(\mathrm{min})}$ coincides with the range of the resolvent $\mathcal{R}_{\lambda}^{(\min )}$ by well-known results on semigroups. Therefore the above result also characterises the domain of the infinitesimal generator of $\mathcal{T}^{(\mathrm{min})}$.

We will give a simpler characterisation of this domain for Markov quantum dynamical semigroups later in Proposition 3.33.

\subsection{Conservativity}

The minimal quantum dynamical semigroup $\mathcal{T}^{(\min )}$ we constructed in Section 3.3 is a quantum subMarkov semigroup.

Let $\mathcal{S}^{(\min )}$ be the predual semigroup on the Banach space of trace class operators in $h$ (see Definition 3.5) of $\mathcal{T}^{(\mathrm{min})}$. The following proposition is immediately proved.

Proposition 3.26 Suppose that the hypothesis A holds. Then, for every $t \geq 0$, the following conditions are equivalent:

1. $\mathcal{T}_{t}^{(\min )}(\mathbb{1})=\mathbb{1}$,

2. $\operatorname{tr}\left(\mathcal{S}_{t}^{(\min )}(\rho)\right)=\operatorname{tr}(\rho)$ for every trace class operator $\rho$ in $h$.

Proof. Notice that

$$
\operatorname{tr}\left(\mathcal{S}_{t}^{(\min )}(\rho)\right)=\operatorname{tr}\left(\mathcal{S}_{t}^{(\min )}(\rho) \mathbb{1}\right)=\operatorname{tr}\left(\rho \mathcal{T}_{t}^{(\min )}(\mathbb{1})\right) .
$$

The equivalence of 1 and 2 is now clear.

In order $\mathcal{T}^{\text {(min) }}$ to be Markov, i.e. conservative or identity preserving, it is necessary that

$$
\langle v, £(\mathbb{1}) u\rangle=\left.\frac{d}{d t}\left\langle v, \mathcal{T}_{t}^{(\min )}(\mathbb{1}) u\right\rangle\right|_{t=0}=0
$$

for every $v, u \in D(G)$. In other words the left-hand side of (3.13) must vanish. We state this property as a stronger form of hypothesis $\mathbf{A}$.

\section{Hypothesis AA}


- the operator $G$ is the infinitesimal generator of a strongly continuous contraction semigroup $\left(P_{t}\right)_{t \geq 0}$ in $\mathcal{H}$,

- the domain of the operators $\left(L_{\ell}\right)_{\ell=1}^{\infty}$ contains the domain of $G$ and, for every $u \in D(G)$ we have

$$
\langle u, G u\rangle+\langle G u, u\rangle+\sum_{\ell=1}^{\infty}\left\langle L_{\ell} u, L_{\ell} u\right\rangle=0 .
$$

This hypothesis unfortunately is not sufficient for the minimal quantum dynamical semigroup to be identity preserving. We shall give some examples later.

In order to study conditions equivalent to conservativity we prove first a useful identity

Proposition 3.27 Suppose that hypothesis AA holds and fix $\lambda>0$. For all $n \geq 1$ we have

$$
\lambda \sum_{k=0}^{n} \mathcal{Q}_{\lambda}^{k}\left(\mathcal{P}_{\lambda}(\mathbb{1})\right)+\mathcal{Q}_{\lambda}^{n+1}(\mathbb{1})=\mathbb{1}
$$

Proof. For all $u \in D(G)$ a standard computation yields

$$
\begin{aligned}
\sum_{\ell=1}^{\infty} \int_{0}^{\infty} \mathrm{e}^{-\lambda t}\left\|L_{\ell} P(t) u\right\|^{2} d t & =-2 \Re e \int_{0}^{\infty} \mathrm{e}^{-\lambda t}\langle P(t) u, G P(t) u\rangle d t \\
& =-\int_{0}^{\infty} \mathrm{e}^{-\lambda t} \frac{d}{d t}\|P(t) u\|^{2} d t \\
& =\|u\|^{2}-\lambda \int_{0}^{\infty} \mathrm{e}^{-\lambda t}\|P(t) u\|^{2} d t
\end{aligned}
$$

Therefore we have

$$
\lambda \mathcal{P}_{\lambda}(\mathbb{1})+\mathcal{Q}_{\lambda}(\mathbb{1})=\mathbb{1} .
$$

This proves (3.29) for $n=0$. Suppose it has been established for an integer $n$. Applying the map $\mathcal{Q}_{\lambda}$ to both sides of (3.29) yields

$$
\lambda \sum_{k=1}^{n+1} \mathcal{Q}_{\lambda}^{k}\left(\mathcal{P}_{\lambda}(\mathbb{1})\right)+\mathcal{Q}_{\lambda}^{n+2}(\mathbb{1})=\mathcal{Q}_{\lambda}(\mathbb{1})=\mathbb{1}-\lambda \mathcal{P}_{\lambda}(\mathbb{1}) .
$$

This proves (3.29) for the integer $n+1$ and completes the proof.

The representation formula (3.26) for the resolvent of the minimal quantum dynamical semigroup allows us to prove a necessary and sufficient condition for conservativity obtained by A.M. Chebotarev in [22] Th. 3.2 (see also [23], [39] Prop. 3.5, 3.6, [26], Prop. 2.7).

Theorem 3.28 Suppose that the hypothesis AA holds and let $\lambda>0$ fixed. Then the following conditions are equivalent: 
1. the minimal quantum dynamical semigroup $\mathcal{T}^{(\min )}$ is Markov,

2. $s-\lim _{n \rightarrow \infty} \mathcal{Q}_{\lambda}^{n}(\mathbb{1})=0$,

3. there exists no non-zero $x \in \mathcal{B}(h)$ such that $\mathcal{Q}_{\lambda}(x)=x$.

Proof. The sequence of positive operators $\left(\mathcal{Q}_{\lambda}^{n}(\mathbb{1})\right)_{n \geq 0}$ is decreasing. In fact (3.29) yields

$$
\mathcal{Q}_{\lambda}^{n}(\mathbb{1})-\mathcal{Q}_{\lambda}^{n+1}(\mathbb{1})=\lambda \mathcal{Q}_{\lambda}\left(\mathcal{P}_{\lambda}(\mathbb{1})\right)
$$

Therefore it is strongly convergent to a positive operator $x$. Letting $n$ tend to $+\infty$ in $(3.29)$, we have

$$
\lambda \mathcal{R}_{\lambda}^{(\min )}(\mathbb{1})+x=\mathbb{1}
$$

Now condition 1 can be alternativcly stated as: $\lambda \mathcal{R}_{\lambda}^{(\min )}(\mathbb{1})$ coincides with $\mathbb{1}$. Therefore the equivalence of conditions 1 and 2 follows.

Condition 2 implies condition 3. In fact, for every $x \in \mathcal{B}(h)$, which is a fixed point for the map $\mathcal{Q}_{\lambda}$ we have

$$
\mathcal{Q}_{\lambda}\left(x^{*}\right)=\left(\mathcal{Q}_{\lambda}(x)\right)^{*}=x^{*} .
$$

Therefore both $x+x^{*}$ and $-i\left(x-x^{*}\right)$ are fixed point for the map $\mathcal{Q}_{\lambda}$. Applying $\mathcal{Q}_{\lambda}^{n}$ to the inequalities

$$
-2\|x\|_{\infty} \mathbb{1} \leq x+x^{*} \leq 2\|x\|_{\infty} \mathbb{1}, \quad-2\|x\|_{\infty} \mathbb{1} \leq i\left(x-x^{*}\right) \leq 2\|x\|_{\infty} \mathbb{1},
$$

we have

$$
\begin{aligned}
& -2\|x\|_{\infty} \mathcal{Q}_{\lambda}^{n}(\mathbb{1}) \leq x+x^{*} \leq 2\|x\|_{\infty} \mathcal{Q}_{\lambda}^{n}(\mathbb{1}), \\
& -2\|x\|_{\infty} \mathcal{Q}_{\lambda}^{n}(\mathbb{1}) \leq i\left(x-x^{*}\right) \leq 2\|x\|_{\infty} \mathcal{Q}_{\lambda}^{n}(\mathbb{1}) .
\end{aligned}
$$

Letting $n$ tend to infinity we obtain $x=0$.

Finally condition 3 implies condition 2 since, if the decreasing sequence of operators $\left(\mathcal{Q}_{\lambda}^{n}(\mathbb{1})\right)_{n \geq 0}$ converges strongly to a non-zero operator $x$, then we have

$$
x=\mathrm{s}-\lim _{n \rightarrow \infty} \mathcal{Q}_{\lambda}^{n}(\mathbb{1})=\mathrm{w}-\lim _{n \rightarrow \infty} \mathcal{Q}_{\lambda}\left(\mathcal{Q}_{\lambda}^{n}(\mathbb{1})\right)=\mathcal{Q}_{\lambda}\left(\mathrm{w}-\lim _{n \rightarrow \infty} \mathcal{Q}_{\lambda}^{n}(\mathbb{1})\right)=\mathcal{Q}_{\lambda}(x)
$$

Therefore condition 3 does not hold.

We give now an example in which the inequality $\mathcal{T}_{t}^{(\min )}(\mathbb{1})<\mathbb{1}$ holds for some $t>0$. This establishes a relationship with a problem in classical probability: escape at infinity in finite time of trajectories of a continuous time Markov process. This example, which is a generalization of Example 3.3 p. 174 in [32], was essentially considered in [38] Sect. 2.

Example 3.4 Let $h$ be the Hilbert space $l^{2}(N)$ of square-summable complexvalued sequences and let $(\lambda(n))_{n \geq 0}$ be a complex sequence such that $\lambda(n) \neq 0$ for every $n \geq 0$. Let $S$ be the right shift operator on $h$ defined on the canonical 
orthonormal basis $\left(e_{n}\right)_{n \geq 0}$ by $S e_{n}=e_{n+1}$, for $n \geq 0$. Consider the operator $\lambda(N)$ in $h$ defined by

$$
D(\lambda(N))=\left\{\left.u \in h\left|\sum_{n \geq 0}\right| \lambda(n)\right|^{2}\left|u_{n}\right|^{2}<\infty\right\}, \quad \lambda(N) u=\sum_{n \geq 0} \lambda(n) u_{n} e_{n}
$$

Let $G$ and $L_{1}$ be the operators with domain $D\left(|\lambda(N)|^{2}\right)$

$$
G=-\frac{1}{2}|\lambda(N)|^{2}, \quad L_{1}=S \lambda(N)
$$

and let $L_{\ell}=0$ for every $\ell \geq 2$. Since $S$ is an isometry the hypothesis AA holds.

A straightforward computation shows that the form $\mathcal{E}(x)$ given by (3.14) applicel to a multiplication operator $M(f)$ by a function $f$ on $I N$ yields

$$
£(M(f))=M(A f)
$$

where

$$
(A f)(n)=|\lambda(n)|^{2}(f(n+1)-f(n)) .
$$

Thus $A$ coincides (on "good" functions) with the infinitesimal generator of a classical pure-birth process.

Let $\mathcal{T}^{\text {(min) }}$ be the minimal quantum dynamical semigroup associated with $G$ and $L$.

In this case we can compute explicitly the operators $\mathcal{Q}_{1}^{n}(\mathbb{1})$. In fact, for every $n, m \geq 0$ and every $k \geq 1$ we have

$$
\left\langle e_{m}, \mathcal{Q}_{1}^{k}(\mathbb{1}) e_{n}\right\rangle=\left\langle e_{m}, e_{n}\right\rangle \prod_{j=0}^{k-1} \frac{|\lambda(n+j)|^{2}}{1+|\lambda(n+j)|^{2}} .
$$

This can be proved by induction. Formula (3.30) is true for $k=1$ because

$$
\begin{aligned}
\left\langle e_{m}, \mathcal{Q}_{1}(\mathbb{1}) e_{n}\right\rangle & =\left\langle e_{m}, e_{n}\right\rangle \int_{0}^{\infty} \mathrm{e}^{-s}|\lambda(n)|^{2} \exp \left(-|\lambda(n)|^{2} s\right) d s \\
& =\left\langle e_{m}, e_{n}\right\rangle \frac{|\lambda(n)|^{2}}{1+|\lambda(n)|^{2}} .
\end{aligned}
$$

A similar explicit computation allows us to complete the induction argument.

We can now give a necessary' and sufficient condition on the sequence

$$
(\lambda(n))_{n \geq 0}
$$

in order the minimal quantum dynamical semigroup $\mathcal{T}^{\text {(min) }}$ to be identity preserving.

Proposition 3.29 The following conditions are equivalent:

1. $\mathcal{T}_{t}^{(\min )}(\mathbb{1})=\mathbb{1}$ for every $t \geq 0$, 
2. the series $\sum_{n \geq 0}|\lambda(n)|^{-2}$ diverges.

Proof. By virtue of Theorem 3.28 it suffices to show that condition 2 is equivalent to

$$
\lim _{k \rightarrow \infty} \prod_{j=0}^{k-1} \frac{|\lambda(n+j)|^{2}}{1+|\lambda(n+j)|^{2}}=0
$$

for every $n \geq 0$.

Due to the inequality

$$
\prod_{j=0}^{k} \frac{|\lambda(n+j)|^{2}}{1+|\lambda(n+j)|^{2}}=\prod_{j=0}^{k} \frac{1}{1+|\lambda(n+j)|^{-2}} \leq\left(1+\sum_{j=0}^{k} \frac{1}{|\lambda(n+j)|^{2}}\right)^{-1},
$$

the limit (3.31) vanishes if condition 2 holds.

Conversely, if the series $\sum_{n>0}|\lambda(n)|^{-2}$ converges, then using the elementary inequality $\log (1+x) \leq x(x \geq \overline{0})$, we have

$$
\begin{aligned}
\log \left(\prod_{j=0}^{k} \frac{|\lambda(n+j)|^{2}}{1+|\lambda(n+j)|^{2}}\right) & =-\sum_{j=0}^{k} \log \left(1+|\lambda(n+j)|^{-2}\right) \\
& \geq-\sum_{j=0}^{k}|\lambda(n+j)|^{-2}
\end{aligned}
$$

Therefore

$$
\lim _{k \rightarrow \infty} \log \left(\prod_{j=0}^{k} \frac{|\lambda(n+j)|^{2}}{1+|\lambda(n+j)|^{2}}\right)>-\infty
$$

and so (3.31) does not hold.

It is worth noticing here that divergence of the series $\sum_{n>0}|\lambda(n)|^{-2}$ is equivalent to non-explosion (i.e. non-escape at infinity in finite time) of a classical pure-birth process with birth intensities $\left(|\lambda(n)|^{2}\right)_{n \geq 0}$ (see [51]).

Here the minimal quantum dynamical semigroup $\mathcal{T}^{(\min )}$ preserves the identity operator $\mathbb{1}$ if and only if this "associated" (in a sense that will be made precise later) classical stochastic process enjoys the above regularity property.

In order to give another necessary and sufficient condition for the minimal quantum dynamical semigroup to be Markov we study the linear manifold

$$
\mathcal{A}_{\lambda}=\left\{x \in \mathcal{B}(h) \mid \mathcal{Q}_{\lambda}(x)=x\right\} .
$$

Clearly $\mathcal{A}_{\lambda}$ is closed for the $\sigma$-weak topology. Moreover we have the following characterisation (see [19], Th. 2.4 p. 92) 
Proposition 3.30 Suppose that the hypothesis AA holds and let $\lambda>0$ fixed. Then, for all $x \in \mathcal{B}(h)$, we have $£(x)=\lambda x$ if and only if $\mathcal{Q}_{\lambda}(x)=x$. In particular

$$
\mathcal{A}_{\lambda}=\{x \in \mathcal{B}(h) \mid £(x)=\lambda x\}
$$

Proof. Let $x$ be an element of $\mathcal{B}(h)$ such that $£(x)=\lambda x$. Then, for every $u, v \in D(G)$ and every $t \geq 0$, we have

$$
\begin{aligned}
\sum_{\ell=1}^{\infty}\left\langle L_{\ell} P(t) v, x L_{\ell} P(t) u\right\rangle & =\lambda\langle P(t) v, x P(t) u\rangle \\
& -\langle G P(t) v, x P(t) u\rangle-\langle P(t) v, x G P(t) u\rangle .
\end{aligned}
$$

Taking Laplace transform of both sides in this identity we obtain

$$
\left\langle v, \mathcal{Q}_{\lambda}(x) u\right\rangle=-\int_{0}^{\infty} \frac{d}{d t} \exp (-\lambda t)\langle P(t) v, x P(t) u\rangle d t=\langle v, x u\rangle
$$

for every $u, v \in D(G)$ and hence, by density of $D(G)$ in $h$, for every $u, v \in h$. Thus $\mathcal{Q}_{\lambda}(x)=x$.

Conversely we now show that the relation $£(x)=\lambda x$ holds in the form sense if $\mathcal{Q}_{\lambda}(x)=x$.

Let $R(\lambda / 2 ; G)$ be the resolvent operator $(\lambda / 2-G)^{-1}$. Notice that, for all $\ell \geq$ 1 , the operator $L_{\ell} R(\lambda / 2 ; G)$ has a bounded extension because of (3.28) and the fact that $G R(\lambda / 2 ; G)$ can be extended to the bounded operator $(\lambda / 2) R(\lambda / 2 ; G)$ $\mathbb{1}$. Hence, using the well-known properties of resolvent operators, we can easily compute the derivative

$$
\begin{aligned}
\frac{d}{d t} L_{\ell} P(t) R(\lambda / 2 ; G) u & =\left(L_{\ell} R(\lambda / 2 ; G)\right) \frac{d}{d t} P(t) u \\
& =-L_{\ell} P(t) u+\frac{\lambda}{2} L_{\ell} P(t) R(\lambda / 2 ; G) u
\end{aligned}
$$

for $u \in D(G)$. For every $u, v \in D(G)$, letting $v^{\prime}=R(\lambda / 2 ; G) v, u^{\prime}=R(\lambda / 2 ; G) u$, since $G R(\lambda / 2 ; G)=(\lambda / 2) R(\lambda / 2 ; G)-\mathbb{1}$, we then have

$$
\begin{aligned}
& \sum_{\ell=1}^{\infty}\left\langle L_{\ell} v^{\prime}, x L_{\ell} u^{\prime}\right\rangle \\
= & -\sum_{\ell=1}^{\infty} \int_{0}^{\infty} \frac{d}{d t}\left(\exp (-\lambda t)\left\langle L_{\ell} P(t) v^{\prime}, x L_{\ell} P(t) u^{\prime}\right\rangle\right) d t \\
= & \sum_{\ell=1}^{\infty} \int_{0}^{\infty} \mathrm{e}^{-\lambda t}\left(\left\langle L_{\ell} P(t) v, x L_{\ell} P(t) u^{\prime}\right\rangle+\left\langle L_{\ell} P(t) v^{\prime}, x L_{\ell} P(t) u\right\rangle\right) d t
\end{aligned}
$$

Therefore, since $\mathcal{Q}_{\lambda}(x)=x$, we obtain

$$
\begin{aligned}
\sum_{\ell=1}^{\infty}\left\langle L_{\ell} v^{\prime}, x L_{\ell} u^{\prime}\right\rangle & =\left\langle v, \mathcal{Q}_{\lambda}(x) u^{\prime}\right\rangle+\left\langle v^{\prime}, \mathcal{Q}_{\lambda}(x) u\right\rangle \\
& =+\left\langle((\lambda / 2) \mathbb{1}-G) v^{\prime}, x u^{\prime}\right\rangle+\left\langle v^{\prime}, x((\lambda / 2) \mathbb{1}-G) u^{\prime}\right\rangle .
\end{aligned}
$$


This shows that $£(x)=\lambda x$ in the form sense because $u^{\prime}, v^{\prime}$ can be chosen arbitrarily in $D(G)$.

The following necessary and sufficient condition for conservativity ([22] Th. 3.3 ) is now obvious.

Proposition 3.31 Suppose that the hypothesis AA holds and let $\lambda>0$ fixed. The following conditions are equivalent:

1. the minimal quantum dynamical semigroup $\mathcal{T}^{(\min )}$ is Markov,

2. there exists no non-zero $x \in \mathcal{B}(h)$ such that $£(x)=\lambda x$.

Proof. Indeed conditions 1 and 2 are equivalent to $\mathcal{A}_{\lambda}=\{0\}$ by Proposition 3.30 and Theorem 3.28.

We finish this section with two properties of Markov minimal quantum dynamical semigroups.

The first one is a result due to E.B. Davies (see [32] Th. 3.2 p.174) giving another necessary and sufficient condition for the minimal quantum dynamical semigroup $\mathcal{T}^{(\mathrm{min})}$ to be Markov.

Proposition 3.32 Suppose that the hypothesis AA holds. The linear manifold $\mathcal{V}$ generated by the rank-one operators

$$
|u\rangle\langle v|, \quad u, v \in D(G)
$$

is contained in the domain of the infinitesimal generator $\mathcal{L}_{*}$ of the predual semigroup $\mathcal{S}^{(\mathrm{min})}$ of $\mathcal{T}^{(\mathrm{min})}$ and

$$
\mathcal{L}_{*}(|u\rangle\langle v|)=|G u\rangle\left\langle v\left|+\sum_{\ell=1}^{\infty}\right| L_{\ell} u\right\rangle\left\langle L_{\ell} v|+| u\right\rangle\langle G v|
$$

(where the series converges in trace norm). Moreover the following conditions are equivalent:

1. the linear manifold $\mathcal{V}$ is a core for $\mathcal{L}_{*}$,

2. the minimal quantum dynamical semigroup $\mathcal{T}^{(\mathrm{min})}$ is Markov.

Proof. For every $u, v \in D(G)$ and every $x \in \mathcal{B}(h)$ equation (3.15) can be written as

$$
\operatorname{tr}\left(x \mathcal{S}_{t}^{(\min )}(|u\rangle\langle v|)\right)=\operatorname{tr}(x|u\rangle\langle v|)+\int_{0}^{t}\left\langle v, £\left(\mathcal{T}_{s}^{(\min )}(x)\right) u\right\rangle d s .
$$

Therefore we have

$$
\begin{array}{r}
\frac{1}{t} \operatorname{tr}\left(x\left(\mathcal{S}_{t}^{(\min )}(|u\rangle\langle v|)-|u\rangle\langle v|\right)\right)=\frac{1}{t} \int_{0}^{t}\left(\left\langle G v, \mathcal{T}_{s}^{(\min )}(x) u\right\rangle\right. \\
\left.+\sum_{\ell=1}^{\infty}\left\langle L_{\ell} v, \mathcal{T}_{s}^{(\min )}(x) L_{\ell} u\right\rangle+\left\langle v, \mathcal{T}_{s}^{(\min )}(x) G u\right\rangle\right) d s
\end{array}
$$


The functions

$$
\begin{aligned}
s & \rightarrow\left\langle G v, \mathcal{T}_{s}^{(\min )}(x) u\right\rangle, \\
s & \rightarrow\left\langle v, \mathcal{T}_{s}^{(\min )}(x) G u\right\rangle, \\
s & \rightarrow\left\langle L_{\ell} v, \mathcal{T}_{s}^{(\min )}(x) L_{\ell} u\right\rangle, \quad \ell \geq 1,
\end{aligned}
$$

are clearly continuous. Moreover, by virtue of (3.28), we have the estimate

$$
\left|\left\langle L_{\ell} v, \mathcal{T}_{s}^{(\min )}(x) L_{\ell} u\right\rangle\right| \leq\|x\| \cdot\left\|L_{\ell} v\right\| \cdot\left\|L_{\ell} u\right\|
$$

for every $\ell \geq 1$. Notice that the series

$$
\begin{aligned}
\sum_{\ell=1}^{\infty}\left\|L_{\ell} v\right\| \cdot\left\|L_{\ell} u\right\| & \leq\left(\sum_{\ell=1}^{\infty}\left\|L_{\ell} v\right\|^{2}\right)^{1 / 2} \cdot\left(\sum_{\ell=1}^{\infty}\left\|L_{\ell} u\right\|^{2}\right)^{1 / 2} \\
& =(-2 \Re e\langle v, G v\rangle)^{1 / 2} \cdot(-2 \Re e\langle u, G u\rangle)^{1 / 2}
\end{aligned}
$$

converge. Hence the function

$$
s \rightarrow \sum_{\ell=1}^{\infty}\left\langle L_{\ell} v, \mathcal{T}_{s}^{(\min )}(x) L_{\ell} u\right\rangle
$$

is continuous by Lebesgue's theorem (applied to the counting measure on $\mathbb{N}$ ). Letting $t$ tend to 0 in (3.33) we have then

$$
\lim _{t \rightarrow 0^{+}} \frac{1}{t} \operatorname{tr}\left(x\left(\mathcal{S}_{t}^{(\min )}(|u\rangle\langle v|)-|u\rangle\langle v|\right)\right)=\langle v, £(x) u\rangle .
$$

Therefore, since the strong generator and the weak generator of $\mathcal{S}^{\text {(min) }}$ coincide (see, for example, [76] Th.1.3 p.43), the rank-one operator $|u\rangle\langle v|(u, v \in D(G)$ ) belongs to the domain of $\mathcal{L}_{*}$ and (3.32) holds (the series is convergent in trace norm).

We show now the equivalence of conditions 1 and 2 .

Suppose that $\mathcal{T}^{(\mathrm{min})}$ is Markov. Since $\mathcal{V}$ is trace-norm dense in the Banach space of trace class operators, $\mathcal{V}$ is a core for $\mathcal{L}_{*}$ if and only if the orthogonal in $\mathcal{B}(h)$ of the linear manifold $\left(\lambda-\mathcal{L}_{*}\right)(\mathcal{V})$ is trivial for some $\lambda>0$ (see, for example, [33], Prop. 3.1 p. 17 or [62] Problem 5.19 p. 166). If $x$ is an element of this orthogonal complement we have

$$
\operatorname{tr}\left(\left(\left(\lambda-\mathcal{L}_{*}\right)(|u\rangle\langle v|)\right) x\right)=0
$$

i.e. $E(x)=\lambda x$ in the form sense. Hence $x=0$ by Proposition 3.31 because $\mathcal{T}^{(\min )}$ is Markov.

Conversely notice that for every $\rho \in \mathcal{V}$ we have

$$
\operatorname{tr}\left(\mathcal{L}_{*}(\rho)\right)=0 .
$$


Therefore, since $\mathcal{V}$ is a core for $\mathcal{L}_{*}$ this identity holds for all $\rho$ in the domain of $\mathcal{L}_{*}$. For all such $\rho$ we have then

$$
\frac{d}{d t} \operatorname{tr}\left(\mathcal{S}_{t}^{(\min )}(\rho)\right)=\operatorname{tr}\left(\mathcal{L}_{*}\left(\mathcal{S}_{t}^{(\min )}(\rho)\right)\right)=0
$$

This shows that $\operatorname{tr}\left(\mathcal{S}_{t}^{(\min )}(\rho)\right)=\operatorname{tr}(\rho)$ for every $t \geq 0$ and every trace class operator $\rho$ because $\mathcal{V}$ is dense with respect to the trace norm. Thus $\mathcal{T}^{(\min )}$ is Markov by Proposition 3.26.

The second one is a useful characterisation of the domain obtained in [45] Lemma 1.1. This is the counterpart at the level of the infinitesimal generator of a well-known property of weak*-continuous semigroups (see [21] Prop. 3.1.23 p.182). Namely, in the present case, an operator $x \in \mathcal{B}(h)$ belongs to the domain of the infinitesimal generator $\mathcal{L}$ of a quant um dynamical semigroup $\mathcal{T}$ if and only if

$$
\sup _{t \geq 0} t^{-1}\left\|\mathcal{T}_{t}(x)-x\right\|_{\infty}<\infty .
$$

Proposition 3.33 Suppose that the hypothesis AA holds and that the minimal quantum dynamical semigroup is Markov. Then the domain of the infinitesimal generator $\mathcal{L}^{(\mathrm{min})}$ of $\mathcal{T}^{(\mathrm{min})}$ is given by all elements $x \in \mathcal{B}(h)$ such that the sesquilinear form $\mathcal{E}(x)$ on $D(G) \times D(G)$

$$
(v, u) \rightarrow\langle v, E(x) u\rangle
$$

is norm-continuous.

Proof. Let $\mathcal{L}_{*}^{(\min )}$ be the infinitesimal generator of the predual semigroup) $\mathcal{S}^{(\mathrm{min})}$. Since $\mathcal{L}^{(\mathrm{min})}=\left(\mathcal{L}_{*}^{(\mathrm{min})}\right)^{*}$, an operator $x \in \mathcal{B}(h)$ belongs to the domain of $\mathcal{L}$ if and only if the linear form on $D\left(\mathcal{L}_{*}^{(\min )}\right)$

$$
\rho \rightarrow \operatorname{tr}\left(\mathcal{L}_{*}^{(\min )}(\rho) x\right)
$$

is continuous for the trace norm $\|\cdot\|_{1}$. Therefore the proof consists essentially in establishing the equivalence of this property with the norm continuity of (3.34).

Clearly the continuity is a necessary condition for $x$ being an element of $D\left(\mathcal{L}^{(\min )}\right)$ because

$$
\begin{aligned}
|\langle v, \mathcal{E}(x) u\rangle| & =\left|\left\langle v \cdot \mathcal{L}^{(\min )}(x) u\right\rangle\right| \\
& \leq\left\|\mathcal{L}^{(\min )}(x)\right\|_{\infty} \cdot\|v\| \cdot\|u\| \\
& =\left\|\mathcal{L}^{(\min )}(x)\right\|_{\infty} \cdot \||u\rangle\left\langle v \|_{1} .\right.
\end{aligned}
$$

We prove now that it is also sufficient. Notice first that, if the sesquilinear form (3.34) is norm continuous, then there exists an operator $y \in \mathcal{B}(h)$ such that

$$
\langle v, £(x) u\rangle=\langle v, y u\rangle
$$


for each $v, u \in D(G)$. Any $\rho \in \mathcal{V}$ can be written as a finite sum

$$
\sum_{j=1}^{d}\left|u_{j}\right\rangle\left\langle v_{j}\right|
$$

where $\left(u_{j}\right)_{1 \leq j \leq d},\left(v_{j}\right)_{1 \leq j \leq d}$ are elements of $D(G)$. We have then

$\left|\operatorname{tr}\left(\mathcal{L}_{*}^{(\min )}(\rho) x\right)\right|=\left|\sum_{j=1}^{d}\left\langle v_{j}, \mathcal{E}(x) u_{j}\right\rangle\right|=\left|\sum_{j=1}^{d}\left\langle v_{j}, y u_{j}\right\rangle\right|=|\operatorname{tr}(\rho y)| \leq\|y\|_{\infty} \cdot\|\rho\|_{1}$,

whenever $x \in \mathcal{B}(h)$ is chosen so that the form (3.34) is continuous. This incrmality also holds for $\rho \in D\left(\mathcal{L}_{*}^{(\min )}\right)$ because the linear manifold $\mathcal{V}$ generated 1 operators $|u\rangle\langle v|$ with $u, v \in D(G)$ is a core for $\mathcal{L}_{*}^{(\min )}$ by Proposition 3.32. Therefore $x$ belongs to the domain of $\mathcal{L}^{(\mathrm{min})}$.

\subsection{Sufficient conditions for conservativity}

The necessary and sufficient conditions for conservativity that were proved in the previous section turn out to be applicable only in some simple cases when, due to the structure of the operators $G$ and $L$ and to a symmetry or an invariance of the form $£$, the minimal quantum dynamical semigroup $\mathcal{T}^{(\mathrm{min})}$ leaves invariant an abelian subalgebra of $\mathcal{B}(h)$ containing the unit $\mathbb{1}$. In these cases (see, for example, [19], [27], [56]) it suffices to study the problem for the classical subMarkov semigroup on the invariant abelian subalgebra.

In this section we give a simple general sufficient condition for the minimal quantum dynamical semigroup to be Markov obtained by A.M. Chebotarev and the author [28].

The starting point of our analysis is a simple remark which is an immediate consequence of Theorem 3.28. The minimal quantum dynamical semigroup $\mathcal{T}^{(\min )}$ is Markov whenever, for a fixed $\lambda>0$, the series

$$
\sum_{k=1}^{\infty}\left\langle u, \mathcal{Q}_{\lambda}^{k}(\mathbb{1}) u\right\rangle
$$

is convergent for all $u$ in a dense subspace of $h$. In fact, in this case, condition 2 of Theorem 3.28 holds. Indeed convergence of the series (3.36) implies that the sequence $\left(\left\langle u, \mathcal{Q}_{\lambda}^{k}(\mathbb{1}) u\right\rangle\right)_{k \geq 1}$ vanishes as $k$ goes to infinity.

In order to find easily verifiable conditions on the operators $G, L_{\ell}$ that guarantee convergence of (3.36), we start by proving an easy estimate.

Let $R(n ; G)$ denote the resolvent operator $(n \mathbb{1}-G)^{-1}$. We can define bounded operators in $h$ formally written as

$$
\sum_{\ell=1}^{\infty}\left(n L_{\ell} R(n ; G)\right)^{*}\left(n L_{\ell} R(n ; G)\right)
$$


by (3.28). In fact we have

$$
\begin{aligned}
\left|\sum_{\ell=1}^{\infty}\left\langle n L_{\ell} R(n ; G) u, n L_{\ell} R(n ; G) u\right\rangle\right| & \leq 2|\langle n R(n ; G) u, n G R(n ; G) u\rangle| \\
& \leq 2\|n R(n ; G) u\| \cdot\|n G R(n ; G) u\| \\
& \leq 4 n\|u\|^{2}
\end{aligned}
$$

for every $u \in D(G)$ by well-known properties of resolvent operators. Thus we denote by $F_{n}$ the unique bounded positive operator satisfying

$$
\sum_{\ell=1}^{\infty}\left\langle n L_{\ell} R(n ; G) u, n L_{\ell} R(n ; G) u\right\rangle=\left\langle u, F_{n} u\right\rangle .
$$

We can prove the following

Proposition 3.34 Suppose that the hypothesis AA holds. For every $u \in h$ we have

$$
\sum_{k=1}^{\infty}\left\langle u, \mathcal{Q}_{\lambda}^{k}(\mathbb{1}) u\right\rangle \leq \liminf _{n \rightarrow \infty}\left\langle u, \mathcal{R}_{\lambda}^{(\min )}\left(F_{n}\right) u\right\rangle .
$$

Proof. For $u \in D(G), n \geq 1$, we have

$$
\begin{aligned}
\left\langle u, \mathcal{P}_{\lambda}\left(F_{n}\right) u\right\rangle & =\sum_{\ell=1}^{\infty} \int_{0}^{\infty} \mathrm{e}^{-\lambda t}\left\|n L_{\ell} R(n ; G) P(t) u\right\|^{2} d t \\
& =\sum_{\ell=1}^{\infty} \int_{0}^{\infty} \mathrm{e}^{-\lambda t}\left\|L_{\ell} P(t)(n R(n ; G) u)\right\|^{2} d t \\
& =\left\langle n R(n ; G) u, n \mathcal{Q}_{\lambda}(\mathbb{1}) R(n ; G) u\right\rangle .
\end{aligned}
$$

Therefore we have

$$
\mathcal{P}_{\lambda}\left(F_{n}\right)=n^{2} R\left(n ; G^{*}\right) \mathcal{Q}_{\lambda}(\mathbb{1}) R(n ; G) .
$$

It follows that the sequence $\left(\mathcal{P}_{\lambda}\left(F_{n}\right)\right)_{n \geq 1}$ is uniformly bounded and converges strongly to $\mathcal{Q}_{\lambda}(\mathbb{1})$ by well-known properties of resolvent operators.

Since the maps $\mathcal{Q}_{\lambda}^{k}$ are normal, we can use the representation (3.26) of the resolvent $\mathcal{R}^{(\mathrm{min})}$ and Fatou's Lemma to get

$$
\begin{aligned}
\sum_{k=1}^{\infty}\left\langle u, \mathcal{Q}_{\lambda}^{k}(\mathbb{1}) u\right\rangle & \leq \liminf _{n \rightarrow \infty} \sum_{k=0}^{\infty}\left\langle u, \mathcal{Q}_{\lambda}^{k}\left(\mathcal{P}_{\lambda}\left(F_{n}\right)\right) u\right\rangle \\
& =\liminf _{n \rightarrow \infty}\left\langle u, \mathcal{R}_{\lambda}^{(\min )}\left(F_{n}\right) u\right\rangle
\end{aligned}
$$

for every $u \in h$. This completes the proof. 
In view of the above proposition it is now clear that the series (3.36) converges whenever we can control $\mathcal{R}_{\lambda}^{(\min )}\left(F_{n}\right)$. This can be done, for example, whenever we can find a positive self-adjoint operator $C$ such that

$$
F_{n} \leq C_{\varepsilon}, \quad \text { and } \quad \sup _{n \geq 1}\left\langle u, \mathcal{R}_{\lambda}^{(\min )}\left(F_{n}\right) u\right\rangle \leq \sup _{\varepsilon>0}\left\langle u, \mathcal{R}_{\lambda}^{(\min )}\left(C_{\varepsilon}\right) u\right\rangle<\infty
$$

for $u$ in a dense subset in $h$, where $C_{\varepsilon}$ are the bounded approximations

$$
C_{\varepsilon}=C(\mathbb{1}+\varepsilon C)^{-1} .
$$

In order to estimate $\left\langle u, \mathcal{R}_{\lambda}^{(\min )}\left(C_{\varepsilon}\right) u\right\rangle$ we introduce our main assumption:

Hypothesis $\mathrm{C}$ - Let $G, L_{\ell}(\ell \geq 1)$ be operators satisfying the hypothesis AA. The operators satisfy the hypothesis $\mathrm{C}$ if there exist a positive self-adjoint operator $C$ and a linear manifold $D$ with the following properties

- $D$ is contained in the domain of $G$,

- $D$ is a core for $C^{1 / 2}$,

- $D$ is an invariant domain for the operators $P(t)(t \geq 0)$ and the linear manifold $R(\lambda ; G)(D)$ is contained in the domain of $C^{1 / 2}$ for each $\lambda>0$,

- the linear manifolds $L_{\ell}(R(\lambda ; G)(D)), \ell \geq 1, \lambda>0$ are contained in the domain of $C^{1 / 2}$,

- there exists a positive constant $b$ such that

$$
2 \Re e\left\langle C^{1 / 2} u, C^{1 / 2} G u\right\rangle+\sum_{\ell=1}^{\infty}\left\langle C^{1 / 2} L_{\ell} u, C^{1 / 2} L_{\ell} u\right\rangle \leq b\left\|C^{1 / 2} u\right\|^{2}
$$

for every $u \in R(\lambda ; G)(D)$ for some $\lambda>0$.

It is worth noticing here that, disregarding the technical assumptions, the hypothesis $\mathbf{C}$ heuristically means that there exist a selfadjoint operator $C$ such that $\mathcal{E}(C) \leq b C$.

Remark. Note that the well-known identity $G R(\lambda ; G)=\lambda R(\lambda ; G)$ - $\mathbb{1}$ implies immediately that the linear manifold $G R(\lambda ; G)(D)$ is contained in the domain of $C^{1 / 2}$ therefore $C^{1 / 2} G R(1 ; G) u$ in (3.37) makes sense.

Remark. The above hypothesis $\mathrm{C}$ is a straightforward generalization of hypothesis $\mathrm{C}$ in [28] where $D$ was assumed to be equal to $D(G)$. The results obtained under this modified assumption are the same and the proofs are almost identical.

In our main application here $G$ (resp. $L_{\ell}$ ) will be a second (resp. first) order differential operator in $L^{2}\left(\mathbb{R}^{d} ; \mathbb{C}\right)$ with regular coefficients and $C$ will be the Laplace operator in $L^{2}\left(\mathbb{R}^{d} ; \mathbb{C}\right)$. In this case it will be convenient to take $D=H^{2}\left(\mathbb{R}^{d} ; \mathbb{C}^{\prime}\right)$. 
We prove now some facts following from the hypothesis $\mathrm{C}$. Let $\|\cdot\|_{C}$ denote the graph norm of the domain of $C^{1 / 2}$

$$
\|u\|_{C}^{2}=\|u\|^{2}+\left\|C^{1 / 2} u\right\|^{2}
$$

and let $\|\cdot\|_{C, \infty}$ denote the corresponding operator norm.

Remark. Note that the hypothesis $\mathbf{C}$ implies that $R(\lambda ; G)(D)$ is dense both in $h$ (with the norm $\|\cdot\|$ ) and $D\left(C^{1 / 2}\right.$ ) (with the norm $\|\cdot\|_{C}$ ) for each $\lambda>0$. Indeed, $D$ is a core for $G$ since it is invariant under the operators $P(t)$ (see e.g. [21] Cor. 3.1.7 p. 167). Therefore $R(\lambda ; G)(D)$ is dense in $h$ by [33], Prop. 3.1 p. 17 or [62] Problem 5.19 p. 166). Moreover, if $v$ belongs to the orthogonal of $R(\lambda ; G)(D)$ in $D\left(C^{1 / 2}\right)$, then, for each $u \in D$ we have

$$
0=\langle v, R(\lambda ; G) u\rangle_{C}=\langle v, R(\lambda ; G) u\rangle+\left\langle C^{1 / 2} v, C^{1 / 2} R(\lambda ; G) u\right\rangle .
$$

Thus

$$
\left|\left\langle C^{1 / 2} v, C^{1 / 2} R(\lambda ; G) u\right\rangle\right| \leq\|v\| \cdot\|R(\lambda ; G) u\| .
$$

This shows that $C^{1 / 2} v$ belongs to the domain of $C^{1 / 2}$ and

$$
\left\langle R\left(\lambda ; G^{*}\right)(\mathbb{1}+C) v, u\right\rangle=0 .
$$

Since $D$ is dense in $h$, we find $R\left(\lambda ; G^{*}\right)(\mathbb{1}+C) v=0$ i.e. $(\mathbb{1}+C) v=(\lambda-$ $\left.G^{*}\right) R\left(\lambda ; G^{*}\right)(\mathbb{1}+C) v=0$. Therefore $v=0$ because $\mathbb{1}+C$ has a bounded inverse.

Similar (indeed simpler) arguments show that $R(\lambda ; G)(D)$ is dense also in $h$ for the norm $\|\cdot\|$.

Lemma 3.35 Suppose that the hypothesis $\mathrm{C}$ holds. Then, for each $\lambda>b$, the domain of $C^{1 / 2}$ is invariant under $R(\lambda ; G)$ and we have

$$
\|R(\lambda ; G)\|_{C, \infty} \leq(\lambda-b)^{-1} .
$$

Proof. Let $u$ be an element of $D$ and let $v=\lambda R(\lambda ; G) u$. Then $v \in C^{1 / 2}$ and the inequality (3.37) yields

$$
\begin{aligned}
\left\|C^{1 / 2} u\right\|^{2} & =\left\|C^{1 / 2}\left(\mathbb{1}-\lambda^{-1} G\right) v\right\|^{2} \\
& =\left\|C^{1 / 2} v\right\|^{2}-2 \lambda^{-1} \Re e\left\langle C^{1 / 2} v, C^{1 / 2} G v\right\rangle+\lambda^{-2}\left\|C^{1 / 2} G v\right\|^{2} \\
& \geq\left(1-\lambda^{-1} b\right)\left\|C^{1 / 2} v\right\|^{2} \\
& =\lambda(\lambda-b)\left\|C^{1 / 2} R(\lambda ; G) u\right\|^{2} .
\end{aligned}
$$

The domain $D$ being a core for $C^{1 / 2}$ for each $u \in D\left(C^{1 / 2}\right)$ we have then

$$
\left\|C^{1 / 2} R(\lambda ; G) u\right\| \leq \lambda^{-1 / 2}\left(1-\lambda^{-1} b\right)^{-1 / 2}\left\|C^{1 / 2} u\right\| \leq(\lambda-b)^{-1}\left\|C^{1 / 2} u\right\| .
$$


This proves the invariance of $D\left(C^{1 / 2}\right)$ under $R(\lambda ; G)$ and the inequality (3.38).

Proposition 3.36 Suppose that the hypothesis $\mathbf{C}$ holds. Then $D\left(C^{1 / 2}\right)$ is invariant under $P(t)$ for each $t \geq 0$, and the restrictions of the operators $P(t)$ to $D\left(C^{1 / 2}\right)$ form a strongly continuous semigroup on the Hilbert space $D\left(C^{1 / 2}\right)$ with norm $\|\cdot\|_{C}$. Moreover we have

$$
\|P(t)\|_{C, \infty} \leq \exp (b t) .
$$

Proof. The restriction to $D\left(C^{1 / 2}\right)$ of the resolvents $R(\lambda ; G)$ obviously satisfies the resolvent identity

$$
R(\lambda ; G)-R(\mu ; G)=(\mu-\lambda) R(\lambda ; G) R(\mu ; G)
$$

for cach $\lambda, \mu>b$. Therefore it is a resolvent family in the Hilbert space $D\left(C^{1 / 2}\right)$ with the norm $\|\cdot\|_{C}$.

Moreover $\operatorname{ker}(R(\lambda ; G))=\{0\}$ since $R(\lambda ; G) u=0$ implies $u=(\lambda \mathbb{1}-G) R(\lambda$; $G) u=0$ and $R(\lambda ; G)\left(D\left(C^{1 / 2}\right)\right)$ is dense in $D\left(C^{1 / 2}\right)$ as remarked before. It follows then from [76] Th.9.3 p.39 that the restriction to $D\left(C^{1 / 2}\right)$ of the operators $R(\lambda ; G)$ yields the resolvent family of a unique densely defined closed operator on $D\left(C^{1 / 2}\right)$.

The inequality (3.38) implies (see [76] Cor.3.8 p.12) that the above densely defined closed operator is the infinitesimal generator of a strongly continuous contraction semigroup on the Hilbert space $D\left(C^{1 / 2}\right)$.

Since this semigroup is given by the strong limit in $D\left(C^{1 / 2}\right)$

$$
\lim _{n \rightarrow \infty}\left(\frac{n}{t} R\left(\frac{n}{t} ; G\right)\right)^{n}
$$

(see [76] Th.8.3 p.33) it follows that $D\left(C^{1 / 2}\right)$ is invariant under the operators $P(t)$ and the semigroup on $D\left(C^{1 / 2}\right)$ coincides with the semigroup obtained by restricting the operators $P(t)$ to $D\left(C^{1 / 2}\right)$. Moreover we have

$$
\begin{aligned}
\|P(t)\|_{C, \infty} & =\lim _{n \rightarrow \infty}\left\|((n / t) R(n / t ; G))^{n}\right\|_{C, \infty} \\
& \leq \lim _{n \rightarrow \infty}\left(1-n^{-1} b t\right)^{-n}=\exp (b t) .
\end{aligned}
$$

This completes the proof.

Corollary 3.37 Suppose that the hypothesis $\mathbf{C}$ holds. Then, for each $u \in$ $R(\lambda ; G)(D)$ with $\lambda>0$, the function $t \rightarrow\left\|C^{1 / 2} P(t) u\right\|^{2}$ is differentiable and

$$
\frac{d}{d t}\left\|C^{1 / 2} P(t) u\right\|^{2}=2 \Re e\left\langle C^{1 / 2} P(t) u, C^{1 / 2} G P(t) u\right\rangle .
$$

Proof. For every $\varepsilon>0$ we have

$$
C_{\varepsilon}^{1 / 2} P(t+s) u=C_{\varepsilon}^{1 / 2} P(t) u+\int_{t}^{t+s} C_{\varepsilon}^{1 / 2} P(r) G u d r
$$


for every $s, t \geq 0$ and $u \in R(\lambda ; G)(D)$. Notice that, since $u=R(\lambda ; G) v$ with $v \in D$, then $G u=\lambda R(\lambda ; G) v-v$ belongs to $D\left(C^{1 / 2}\right)$. Moreover, by Proposition 3.36 , the function $t \rightarrow C^{1 / 2} P(t) G u$ is continuous on $[0,+\infty[$ for the norm $\|\cdot\|$ on $h$ and satisfies the inequality $\left\|C^{1 / 2} P(t) G u\right\| \leq \exp (b t)\left\|C^{1 / 2} G u\right\|$. This allows us to let $\varepsilon$ tend to 0 to get

$$
C^{1 / 2} P(t+s) u=C^{1 / 2} P(t) u+\int_{t}^{t+s} C^{1 / 2} P(r) G u d r .
$$

A straightforward computation shows that the difference

$$
\left\|C^{1 / 2} P(t+s) u\right\|^{2}-\left\|C^{1 / 2} P(t) u\right\|^{2}
$$

for $s, t \geq 0$, can be written in the form

$$
\begin{aligned}
& \left\|C^{1 / 2}(P(t+s)-P(t)) u\right\|^{2}+2 \Re e\left\langle C^{1 / 2} P(t) u, C^{1 / 2}(P(t+s)-P(t)) u\right\rangle \\
& =2 \Re e \int_{t}^{t+s}\left\langle C^{1 / 2} P(t) u, C^{1 / 2} P(r) G u\right\rangle d r+\left\|\int_{t}^{t+s} C^{1 / 2} P(r) G u d r\right\|^{2}
\end{aligned}
$$

Therefore the function $t \rightarrow\left\|C^{1 / 2} P(t) u\right\|^{2}$ is absolutely continuous. Moreover the last term is an infinitesimum of order bigger than $s$ as $s$ tends to 0 since

$$
\left\|\int_{t}^{t+s} C^{1 / 2} P(r) G u d r\right\|^{2} \leq s \int_{t}^{t+s}\left\|C^{1 / 2} P(r) G u\right\|^{2} d r .
$$

The proof is now immediate.

Under the hypothesis $\mathbf{C}$ we can prove a useful estimate of $\mathcal{R}_{\lambda}^{(\min )}\left(C_{\varepsilon}\right)$.

Proposition 3.38 Suppose that the hypothesis $\mathbf{C}$ holds. Then, for all $\lambda>b$ and all $u \in D\left(C^{1 / 2}\right)$, we have

$$
(\lambda-b) \sup _{\varepsilon>0}\left\langle u, \mathcal{R}_{\lambda}^{(\min )}\left(C_{\varepsilon}\right) u\right\rangle \leq\left\|C^{1 / 2} u\right\|^{2} .
$$

Proof. Let $\left(\mathcal{R}_{\lambda}^{(n)}\right)_{n>0}$ be the sequence of positive linear maps considered in the proof of Theorem 3.25.

It suffices to show that, for all $n \geq 0, \lambda>b$ and $u \in D\left(C^{1 / 2}\right)$, the operator $\mathcal{R}_{\lambda}^{(n)}\left(C_{\varepsilon}\right)$ satisfies

$$
(\lambda-b) \sup _{\varepsilon>0}\left\langle u, \mathcal{R}_{\lambda}^{(n)}\left(C_{\varepsilon}\right) u\right\rangle \leq\left\|C^{1 / 2} u\right\|^{2} .
$$

The above inequality holds for $n=0$ and $u \in R(\lambda ; G)(D)$. Indeed, since $D$ is invariant under $P(t)$, then $P(t) R(\lambda ; G)(D)=R(\lambda ; G)(P(t)(D)) \subseteq R(\lambda ; G)(D)$ 
is contained in $D\left(C^{1 / 2}\right)$ and, integrating by parts, we have

$$
\begin{aligned}
& \lambda\left\langle u, \mathcal{R}_{\lambda}^{(0)}\left(C_{\varepsilon}\right) u\right\rangle \\
= & \lambda \int_{0}^{\infty} \mathrm{e}^{-\lambda t}\left\langle P(t) u, C_{\varepsilon} P(t) u\right\rangle d t \\
\leq & \lambda \int_{0}^{\infty} \mathrm{e}^{-\lambda t}\left\|C^{1 / 2} P(t) u\right\|^{2} d t \\
= & \left\|C^{1 / 2} u\right\|^{2}+2 \text { re } \int_{0}^{\infty} e^{-\lambda t}\left\langle C^{1 / 2} P(t) u, C^{1 / 2} C P(t) u\right\rangle d t .
\end{aligned}
$$

The inequality (3.37) yields

$$
\begin{aligned}
\lambda\left\langle u, \mathcal{R}_{\lambda}^{(0)}\left(C_{\varepsilon}\right) u\right\rangle & \leq\left\|C^{1 / 2} u\right\|^{2}+b \int_{0}^{\infty},{ }^{-\lambda t}\left\|C^{1 / 2} P(t) u\right\|^{2} d t \\
& \left.=\left\|C^{1 / 2} u\right\|^{2}+b i s u p\right)\left\langle u \cdot \mathcal{R}_{\lambda}^{(0)}\left(C_{\varepsilon}\right) u\right\rangle .
\end{aligned}
$$

This proves (3.40) for $n=0$ and $u \in R(\lambda ; G)(D)$. Since $R(\lambda ; G)(D)$ is dense in $D\left(C^{1 / 2}\right)$ for the norm $\|\cdot\|_{C},(3.40)$ holds also for $n \in D\left(C^{1 / 2}\right)$.

Suppose that $(3.40)$ has been established for an integer $n$ and every $u \in$ $D\left(C^{1 / 2}\right)$. Notice that for every $u \in R\left(\lambda: G(D)\right.$ the vectors $L_{\ell} P(t) u$ belong to $D\left(C^{1 / 2}\right)$. Thus. from the second equation $(3.17)$ (or from $(3.27)$ ) and the definition of $\mathcal{R}_{\lambda}^{(n)}$, we have

$$
\begin{aligned}
& \left\langle u, \mathcal{R}_{\lambda}^{(n+1)}\left(C_{z}\right) u\right\rangle \\
= & \left\langle u, \mathcal{P}_{\lambda}\left(C_{\varepsilon}\right) u\right\rangle+\sum_{\ell=1}^{\infty} \int_{0}^{\infty} e^{-\lambda t}\left\langle L_{\ell} P(t) u, \mathcal{R}_{\lambda}^{(n)}\left(C_{c}\right) L_{\ell} P(t) u\right\rangle d t . \\
\leq & \left\langle u, P_{\lambda}\left(C_{\varepsilon}\right) u\right\rangle+\frac{1}{\lambda-b} \sum_{\ell=1}^{\infty} \int_{0}^{\infty} e^{-\lambda t}\left\|C^{1 / 2} L_{i} P(t) u\right\|^{2} d t .
\end{aligned}
$$

Using the inequality (3.37) and integrating by parts we obtain

$$
\begin{aligned}
\sum_{\ell=1}^{\infty} \int_{0}^{\infty} e^{-\lambda t}\left\|C^{1 / 2} L_{i} P(t) u\right\|^{2} d t & \leq \int_{0}^{\infty} \mathrm{e}^{-\lambda t}\left(-\frac{d}{d t}\left\|C^{1 / 2} P(t) u\right\|^{2}\right) d t \\
& +b \int_{0}^{\infty} \mathrm{e}^{-\lambda t}\left\|C^{1 / 2} P(t) u\right\|^{2} d t \\
& =\left\|C^{1 / 2} u\right\|^{2}-(\lambda-b) \int_{0}^{\infty} \mathrm{e}^{-\lambda t}\left\|C^{1 / 2} P(t) u\right\|^{2} d t \\
& \leq\left\|C^{1 / 2} u\right\|^{2}-(\lambda-b)\left\langle u, \mathcal{P}_{\lambda}\left(C_{\varepsilon}\right) u\right\rangle .
\end{aligned}
$$


Therefore (3.40) for $n+1$ and $u \in R(\lambda ; G)(D)$ follows. Since $R(\lambda ; G)(D)$ is dense in $D\left(C^{1 / 2}\right)$, this completes the proof.

We can now prove the main results of this section.

Theorem 3.39 Suppose that there exists an operator $C$ satisfying hypothesis C such that

$$
\left\langle u, F_{n} u\right\rangle \leq\langle u, C u\rangle
$$

for each $u \in D(C), n \geq 1$. Then the minimal quantum dynamical semigroup is Markov.

Proof. Fix $\lambda>b$. Under the hypotheses of the theorem, for $\varepsilon>0$, the bounded operators $\left(F_{n}\right)_{\varepsilon}$ and $C_{\varepsilon}$ satisfy the inequality $\left(F_{n}\right)_{\varepsilon} \leq C_{\varepsilon}$ (see, for example, [78] Chap. 8, Ex. 51, p.317). Applying Proposition 3.38, we obtain the estimate

$$
\begin{aligned}
\sum_{k=1}^{\infty}\left\langle u, \mathcal{Q}_{\lambda}^{k}(\mathbb{1}) u\right\rangle & \leq \liminf _{n \rightarrow \infty} \sup _{\varepsilon>0}\left\langle u, \mathcal{R}_{\lambda}^{(\min )}\left(\left(F_{n}\right)_{\varepsilon}\right) u\right\rangle \\
& \leq \sup _{\varepsilon>0}\left\langle u, \mathcal{R}_{\lambda}^{(\min )}\left(C_{\varepsilon}\right) u\right\rangle \\
& \leq(\lambda-b)^{-1}\left\|C^{1 / 2} u\right\|^{2}<+\infty
\end{aligned}
$$

for every $u \in D\left(C^{1 / 2}\right)$. Therefore the minimal quantum dynamical semigroup is Markov because condition 2 of Theorem 3.28 is fulfilled.

Remark. Notice that, in the above theorem, we did not assume that the quadratic form

$$
u \rightarrow-2 \Re e\langle u, G u\rangle
$$

with domain $D(G)$ is closable. This phenomenon happens in quite simple and interesting cases as, for example, in Example 5.3 in [28].

Theorem 3.40 Suppose that the hypothesis AA holds and there exists a positive self-adjoint operator $\Phi$ in h such that:

1. the domain of the positive square root $\Phi^{1 / 2}$, contains the domain of $G$ and, for every $u \in D(G)$, we have

$$
-2 \Re e\langle u, G u\rangle=\sum_{\ell=1}^{\infty}\left\langle L_{\ell} u, L_{\ell} u\right\rangle=\left\langle\Phi^{1 / 2} u, \Phi^{1 / 2} u\right\rangle,
$$

2. there exists a positive self-adjoint operator $C$ satisfying the hypothesis $\mathbf{C}$ such that the domain of $C$ is contained in the domain of $\Phi$ and, for every $u \in D(C)$, we have

$$
\left\langle\Phi^{1 / 2} u, \Phi^{1 / 2} u\right\rangle \leq\left\langle C^{1 / 2} u, C^{1 / 2} u\right\rangle
$$


Then the minimal quantum dynamical semigroup is Markov.

Proof. Let $\lambda>b$ and $u \in D$ fixed. For $\varepsilon>0$, the bounded operators $\Phi_{\varepsilon}$ and $C_{\varepsilon}$ satisfy the inequality $\Phi_{\varepsilon} \leq C_{\varepsilon}$ (see [78] Chap. 8, Ex. 51, p.317). Moreover, for $u \in D(G)$, we have

$$
\begin{aligned}
\sup _{\varepsilon>0}\left\langle u, \mathcal{P}_{\lambda}\left(\Phi_{\varepsilon}\right) u\right\rangle & =\int_{0}^{\infty} \mathrm{e}^{-\lambda t}\left\|\Phi^{1 / 2} P(t) u\right\|^{2} d t \\
& =\sum_{\ell=1}^{\infty} \int_{0}^{\infty} \mathrm{c}^{-\lambda t}\left\|L_{\ell} P(t) u\right\|^{2} d t \\
& =\left\langle u, \mathcal{Q}_{\lambda}(\mathbb{1}) u\right\rangle .
\end{aligned}
$$

Therefore the increasing family of operators $\left(\mathcal{P}_{\lambda}\left(\Phi_{\varepsilon}\right)\right)_{\varepsilon>0}$ is uniformly bounded and. since $D(G)$ is dense in $h$, it follows that it converges strongly to $\mathcal{Q}_{\lambda}(\mathbb{1})$ as E gocs to 0 . The maps $\mathcal{Q}_{\lambda}^{k}$ being $\sigma$-weakly continuous, we have

$$
\begin{aligned}
\sum_{k=0}^{\infty}\left\langle u, \mathcal{Q}_{\lambda}^{k+1}(\mathbb{1}) u\right\rangle & =\sup _{\varepsilon>0} \sum_{k=0}^{\infty}\left\langle u, \mathcal{Q}^{k}\left(P_{\lambda}\left(\Phi_{\varepsilon}\right)\right) u\right\rangle \\
& =\sup _{\varepsilon>0}\left\langle u, \mathcal{R}_{\lambda}^{(\min )}\left(\Phi_{\varepsilon}\right) u\right\rangle
\end{aligned}
$$

by Theorem 3.25. ¿From Proposition 3.38 we obtain the estimate

$$
\sum_{k=1}^{\infty}\left\langle u, \mathcal{Q}_{\lambda}^{k}(\mathbb{1}) u\right\rangle \leq \sup _{\varepsilon>0}\left\langle u, \mathcal{R}_{\lambda}^{(\mathrm{min})}\left(C_{\varepsilon}\right) u\right\rangle \leq(\lambda-b)^{-1}\left\|C^{1 / 2} u\right\|^{2}
$$

for every $u \in D\left(C^{1 / 2}\right)$. Therefore the minimal quantum dynamical semigroup is Markov because condition 2 of Theorem 3.28 holds.

It has been shown in [53] that condition $\mathbf{C}$ can be interpreted as an a priori estimate on the minimal quantum subMarkov semigroup associated with the operators $G, L_{\ell}$.

The following corollary gives an easier (but weaker) sufficient condition for conservativity.

Corollary 3.41 Suppose that the hypothesis AA holds and there exists a selfadjoint operator $C$ with domain coinciding with the domain of $G$ and a core $D$ for $C$ with the following properties:

a) $L_{\ell}(D) \subseteq D\left(C^{1 / 2}\right)$ for all $\ell \geq 1$,

b) there exists a self-adjoint operator $\Phi$ such that

$$
-2 \Re e\langle u, G u\rangle=\langle u, \Phi u\rangle \leq\langle u, C u\rangle
$$

for all $u \in D$, 
c) there exists a positive constant $b$ such that the inequality

$$
2 \Re e\langle C u, G u\rangle+\sum_{\ell=1}^{\infty}\left\langle C^{1 / 2} L_{\ell} u, C^{1 / 2} L_{\ell} u\right\rangle \leq b\langle u, C u\rangle
$$

holds for every $u \in D$.

Then the minimal quantum dynamical semigroup is Markov.

Proof. If we consider $D(G)$ and $D(C)$ as Hilbert spaces equipped with their respective graph norms, then it follows readily that the inclusion map form $D(G)$ to $D(C)$ is closed and everywhere defined. An application of the Closed Graph Theorem implies that $G$ is relatively bounded with respect to $C$. Exchanging $G$ and $C$ the same argument shows that also $C$ is relatively bounded with respect to $G$. Thus there exist constants $c_{1}, c_{2}, c_{3}, c_{4}$ such that

$$
\|G u\| \leq c_{1}\|C u\|+c_{2}\|u\|, \quad\|C u\| \leq c_{3}\|G u\|+c_{4}\|u\| .
$$

Therefore $D$ is a core for both $C$ and $G$ and, for each $u \in D(G)=D(C)$, there exists a sequence $\left(u_{n}\right)_{n \geq 1}$ in $D$ such that the sequences $\left(G u_{n}\right)_{n \geq 1}$ and $\left(C u_{n}\right)_{n \geq 1}$ converge strongly to $G u$ and $C u$ respectively.

This remark, together with the hypothesis b), allows to check immediately the hypothesis 1 of Theorem 3.40 and the inequality in the hypothesis 2 of the same theorem.

The Corollary follows if we show that the operator $C$ satisfies the hypothesis $\mathrm{C}$ taking there as domain $D$ the domain of $G$. Indeed this is obviously a core for $C^{1 / 2}$ and it is obviously invariant for the operators $P(t)$ for $t \geq 0$ and $R(\lambda ; G)$ for $\lambda>0$.

Taking sequences $\left(u_{n}\right)_{n \geq 1}$ in $D$ such that $\left(G u_{n}\right)_{n \geq 1}$ and $\left(C u_{n}\right)_{n \geq 1}$ converge strongly to $G u$ and $C u$ we can show that the linear manifolds $L_{\ell}(D(G))$ are contained in the domain of $C^{1 / 2}$ and that the inequality (3.41) holds for all $u \in$ $D(G)$. If, moreover, $u$ belongs to $R(\lambda ; G)(D(G))=D\left(G^{2}\right)$, then the inequality (3.37) follows.

This shows that $C$ satisfies the hypothesis $\mathbf{C}$. 